\title{
An Equilibrium Analysis of Market Selection Strategies and Fee Strategies in Competing Double Auction Marketplaces
}

\author{
Bing Shi · Enrico H. Gerding • \\ Perukrishnen Vytelingum • Nicholas R. Jennings
}

the date of receipt and acceptance should be inserted later

\begin{abstract}
In this paper, we propose a game-theoretic framework for analysing competing double auction marketplaces that vie for traders and make profits by charging fees. Firstly, we analyse the equilibrium strategies for the traders' market selection decision for given market fees using evolutionary game theory. Using this approach, we investigate how traders dynamically change their strategies, and thus, which equilibrium, if any, can be reached. In so doing, we show that, when the same type of fees are charged by two marketplaces, it is unlikely that competing marketplaces will continue to co-exist when traders converge to their equilibrium market selection strategies. Eventually, all the traders will congregate in one marketplace. However, when different types of fees are allowed (registration fees and profit fees), competing marketplaces are more likely to co-exist in equilibrium. We also find that sometimes all the traders eventually migrate to the marketplace that charges higher fees. We then further analyse this phenomenon, and specifically analyse how bidding strategies and random exploration of traders affects this migration respectively. Secondly, we analyse the equilibrium strategies of the marketplaces when they have the ability to vary their fees in response to changes in the traders' market selection strategies. In this case, we consider the competition of the marketplaces as a two-stage game, where the traders' market selection strategies are conditional on the market fees. In particular, we use a coevolutionary approach to analyse how competing marketplaces dynamically set fees while taking into account the dynamics of the traders' market selection strategies. In so doing, we find that two identical marketplaces undercut each other, and they will eventually charge the minimal fee as we set that guarantees positive market profits for them. Furthermore,
\end{abstract}

Bing Shi

School of Electronics and Computer Science, University of Southampton, Southampton, SO17 1BJ, UK

E-mail: bs07r@ecs.soton.ac.uk

Enrico H. Gerding

School of Electronics and Computer Science, University of Southampton, Southampton, SO17 1BJ, UK

E-mail: eg@ecs.soton.ac.uk

Perukrishnen Vytelingum

School of Electronics and Computer Science, University of Southampton, Southampton, SO17 1BJ, UK

E-mail: pv@ecs.soton.ac.uk

Nicholas R. Jennings

School of Electronics and Computer Science, University of Southampton, Southampton, SO17 1BJ, UK

E-mail: nrj@ecs.soton.ac.uk 
we extend the co-evolutionary analysis of the marketplaces' fee strategies to more general cases. Specifically, we analyse how an initially disadvantaged marketplace with an adaptive fee strategy can outperform an initially advantaged one with a fixed fee strategy, or even one with an adaptive fee strategy, and how competing marketplaces evolve their fee strategies when different types of fees are allowed.

Keywords Competing Double Auction Marketplaces · Market Selection Strategy · Fee Strategy $\cdot$ Evolutionary Game Theory $\cdot$ Co-Evolutionary Approach

\section{Introduction}

Exchanges, in which securities, futures, stocks and commodities can be traded, are becoming ever more prevalent. Now, many of these adopt the double auction market mechanism which is a particular type of two-sided marketplace with multiple buyers (one side) and multiple sellers (the other side) (Friedman and Rust, 1993). Specifically, in such a mechanism, traders can submit offers at any time in a specified trading round and they will be matched by the marketplace at a specified time. The advantages of this mechanism are that traders can enter the marketplace at any time and they can trade multiple homogeneous or heterogeneous items in one place without travelling around several marketplaces. In addition, this mechanism is highly efficient in terms of trading goods between buyers and sellers (Smith, 1962). These benefits have led many electronic marketplaces to also use this format. For example, Google offers DoubleClick Ad Exchange (http://www.doubleclick.com), which is a real-time double auction marketplace enabling large online ad publishers, on one side, and ad networks and agencies, on the other side, to buy and sell advertising space. Another example is FastParts (http://www. fastparts. com), which provides a double auction to trade excess electronic components and used manufacturing equipments. However, because of the globalised economy, such marketplaces do not exist in isolation. Thus they compete against each other to attract traders and make profits by charging fees. For example, stock exchanges compete to attract companies to list their stocks in their marketplaces and make profits by charging listing fees to these companies. Google's DoubleClick Ad Exchange competes against other ad exchanges, such as Microsoft's AdECN (http://www.adecn.com) and Yahoo!'s Right Media (http://www.rightmedia.com) in order to attract ad publishers and ad networks and agencies. Thus such inter-marketplace competition is becoming an increasingly important area of research.

Specifically, for such contexts, there exist two key research issues. The first is how the traders select which marketplace to participate in (the market selection strategies). This problem is challenging since this choice not only depends on the fees charged by the marketplaces, but also on so-called network effects, whereby a trader's welfare depends on the number and types of other traders already present. Then, given the traders' strategies for selecting marketplaces, the second issue is how competing marketplaces should set their fees to make profits (the fee strategies). Intuitively, we can see that there exists a conflict between attracting traders and making profits. This is because when the fees are increased to try and increase profits, traders are likely to leave the marketplace which, in turn, will cause a decrease in profits for that marketplace. Thus the competing marketplace has to be able to set appropriate fees to maximise its profit, while at the same time maintain market share at a good level. Against this background, in this paper, we analyse the equilibrium strategies of traders for selecting a marketplace as well as marketplaces for setting fees. The insights from this analysis will be useful to guide the design of a competing marketplace in terms of setting fees. 
In this context, a number of theoretical models have been proposed to analyse competing two-sided marketplaces (see Section 2 for details). However, most of this work only considers the positive network effect (Farrell and Klemperer, 2007; Kartz and Shapiro, 1985), whereby, buyers(sellers) prefer marketplaces which have a larger number of sellers(buyers) since this gives the buyers(sellers) access to more choices. Such an effect will always push all traders towards concentrating into a single marketplace. However, these works usually ignore the internal competition between traders on the same side (i.e. between buyers and between sellers). In practice, traders on one side will typically compete with each other in order to be matched with traders on the other side. ${ }^{1}$ This is also called a negative network effect (Farrell and Klemperer, 2007; Kartz and Shapiro, 1985) where traders prefer marketplaces with fewer other traders on the same side. In our setting we consider double auction marketplaces to match traders to trade goods and set transaction prices (which are affected by demand and supply), and thereby consider both positive and negative network effects. This negative network effect is important because it encourages traders to distribute across different marketplaces, thereby making it more likely for several competing marketplaces to co-exist in the long term. Moreover, most of the existing work on competing two-sided marketplaces assumes that all traders are homogeneous (i.e. have the same preferences), and the marketplaces have complete information about the preferences (also called the types) of traders. In real-world auction marketplaces, however, traders usually have heterogeneous preferences which are privately known. In this case, the traders, in choosing their marketplaces, not only care about the number of other traders, but also their types. Furthermore, in the real world, competing marketplaces may charge different types of fees to make profits, which may have different effects on traders' market selection. Specifically, there exist two types of fees which are usually charged by real-world marketplaces: ex ante fees, which are charged to traders when they enter the marketplace, and ex post fees, which are charged to traders after they make transactions. In our work, we will consider all these factors, and in doing so, are the first to comprehensively analyse competing double auction marketplaces from a theoretical perspective. ${ }^{2}$

In more detail, in this paper, we assume that there are heterogeneous traders with privately known preferences, and consider registration and profit fees as typical examples of $e x$ ante and ex post fees respectively. Based on these assumptions, we theoretically analyse the market selection strategies for traders and the fee strategies for marketplaces. Intuitively, we can see that how a trader selects a marketplace depends on other traders' decisions as well as the marketplaces' fees, and that how a competing marketplace sets its fees depends on traders' market selection decisions and other marketplaces' fees. Thus game theory (Fudenberg and Tirole, 1991), which mathematically studies such strategic interactions between self-interested agents (where an individual's success in making choices depends on the choices of others), is appropriate to be used for analysing our system. However, due to the high complexity of this game with multiple traders and marketplaces and with incomplete information about traders' types, it is infeasible to use traditional game theory to analytically derive the equilibrium strategies. Therefore, in this paper, we adopt evolutionary game theory (EGT) to analyse the equilibrium strategies for traders and marketplaces. This approach was originated by John Maynard-Smith, who applied game theory to biology (Maynard-Smith, 1982). It offers a way to compute the equilibrium strategies for players, and has been widely used to analyse complicated games (e.g. Phelps et al, 2006, 2010; Vytelingum et al, 2008a). Specifically, in this paper, we will use replicator dynamics, which

\footnotetext{
1 For example, in the two-sided marketplace providing dating service for males and females, males (one side) have to compete with each other in order to date (be matched with) females (the other side).

2 There exists some empirical work that also considers these factors (see Section 2).
} 
are used in EGT to control the learning process, to computationally determine the equilibrium strategies. Furthermore, in order to allow for a tractable analysis for this complicated game, we limit the number of trader types (to two). This canonical setting is common in theoretical work in this broad area and allows us to distill the key insights and patterns of behaviour which can then be extended by others.

This paper advances the state-of-the-art in the following ways. Firstly, after proposing a game-theoretic framework, we use evolutionary game theory to compute the Nash equilibrium (the most common solution concept in game theory) market selection strategies for traders given different market fees. We analyse how traders dynamically change their market selection strategies and which of the equilibria can be reached. In doing so, we also investigate whether competing marketplaces can co-exist, and the competition can be maintained, or whether the marketplaces collapse to a monopoly setting where all traders move to one marketplace. This is important since competition drives efficiency and offers more and better choices to traders. We show that, based on the assumptions of our framework, when the same type of fees are charged, it is unlikely that multiple competing marketplaces will co-exist despite the negative network effect; all traders will simply converge to one of the marketplaces in equilibrium. However, when different types of fees are allowed, competing marketplaces can co-exist. Furthermore, we find that all traders may converge to the marketplace that charges higher fees when the marketplace initially has a larger market share. This means that the marketplace can maintain both a high number of traders and high profits. We then analyse this interesting phenomenon in more detail. Specifically, we analyse in what situations traders select the marketplace that charges higher fees and how this selection is affected by traders' bidding behaviour and random exploration. After having established the traders' equilibrium strategies, we proceed to analyse how marketplaces should set their fees to make profits in equilibrium by taking into account the influence of traders' strategies on marketplaces' fee strategies. This is modeled as a two-stage game where, in the first stage, competing marketplaces set their fees and, in the second stage, traders select a marketplace conditional on these fees. We can see that the traders' market selection strategies and the marketplaces' fee strategies are closely intertwined. Thus we use a co-evolutionary approach to analyse this game. We first consider that competing marketplaces charge the same type of fees. Specifically, we find that two initially identical competing marketplaces will eventually charge the minimal fee that guarantees positive market profits for them. Furthermore, we show that an initially disadvantaged marketplace with an adaptive fee strategy can beat the initially advantaged one with a fixed fee strategy. We also find that, by dynamically evolving the fee strategy, it is possible for the marketplace that is initially at a disadvantage to outperform its opponent, even when the opponent is also able to evolve its fee strategy. We then extend the analysis to the case where different types of fees are allowed. We find that one marketplace eventually attracts all traders and then charges a very high fee.

The structure of the rest of the paper is as follows. In Section 2, we discuss related work. In Section 3, we describe the game-theoretic framework. In Section 4, we analyse the traders' equilibrium strategies for market selection for given (fixed) market fees. In Section 5 , we use a co-evolutionary approach to analyse equilibrium fee strategies of competing marketplaces. Finally, we conclude in Section 6.

\section{Related Work}

As mentioned in the introduction, many theoretical models analysing competing two-sided marketplaces only consider the positive network effect, where buyers will prefer market- 
places with more sellers and vice versa for sellers. This gives rise to a "chicken and egg" problem which lies at the core of two-sided marketplaces: to attract buyers (one side), a marketplace should have a large base of the registered sellers (the other side), but these will be willing to register only if they expect many buyers to show up. One of the most important works on this problem is from Caillaud and Jullien (2003), who analysed the competition between two marketplaces. In their work, they assume that traders are homogeneous and the market selection only depends on the number of traders of the other side. For this setting, they analyse a "divide-and-conquer" strategy of subsidizing one side of the traders (by charging negative registration fees) while recovering loss (by charging positive transaction fees) from the other side. They show that, when traders can only enter one marketplace at a time, by adopting the "divide-and-conquer" strategy, in equilibrium, one marketplace will attract all traders, but it has to give up all profit. Another work in this vein is by Damiano and Hao (2008), in which traders select marketplaces only according to the quality characteristics (type) of the other side of traders, instead of the number of traders, and thus they do not consider network effects. Recent work by Lee (2008) analyses under what conditions two competing marketplaces can co-exist. They point out that strong marketplace differentiation or weak positive network effect can make competing marketplaces co-exist. This is consistent with our analysis, where we find that, when competing marketplaces differentiate from one another by charging different types of fees, they may co-exist in equilibrium.

Although the above work is related, none of these papers specifically consider double auction marketplaces to match traders. This changes the problem because, in the competition of double auction marketplaces, the market selection strategy of the traders not only depends on the number of traders choosing the marketplaces, but also on their own types and those of other traders choosing the marketplaces. Furthermore, in addition to the positive network effect, there also exist negative network effects. Competition between two double auction marketplaces is considered by Ellison et al (2004). They show that, in some cases, the negative network effect has a larger impact than the positive network effect, and traders will not migrate from this state, which means that two competing marketplaces can co-exist in equilibrium. This model is similar to ours since we also consider heterogeneous traders and both positive and negative size effects. However, Ellison et al (2004) make the simplifying assumption that traders choose a marketplace before learning their own types, and thus the market selection strategy is independent of a trader's type. Therefore, unlike in our model, using their model, they show that similar marketplaces can co-exist in equilibrium. In contrast, we find that traders will converge to one marketplace except for the case where there is strong market differentiation (i.e. where competing marketplaces charge different types of fees).

The other strand of work that explores this area is primarily related to the Market Design Competition (CAT), which was first introduced as part of the Trading Agent Competition (TAC) in 2007 (Gerding et al, 2007a). This competition considers a complicated setting for competing double auction marketplaces. In more detail, in this competition, entrants need to design effective market policies and set appropriate fees to attract traders and make profits. There are a large number of traders, which have privately known types and can move freely between different marketplaces to choose the one that they think will be most profitable. Intuitively, we can imagine that traders' migration will significantly determine the final competition results. For this setting, Niu et al (2007) provide an experimental analysis of traders' market selection strategies. They show that, when traders are able to explore to search for the most profitable marketplace, they exhibit a strong tendency to converge to the marketplaces charging low fees. They also show that the migration of traders is affected by different exploration algorithms. Then, based on this work, Cai et al (2008) ran experiments 
to analyse the impact of multiple marketplaces on the allocative efficiency. ${ }^{3}$ They show that dividing traders over multiple marketplaces leads to a loss of allocative efficiency, and the loss is reduced when traders are allowed to choose the most profitable marketplace. In addition, a number of entrants have described their specific design of the competing double auction marketplaces for recent years' CAT competition (Vytelingum et al, 2008b; Petric et al, 2008; Stavrogiannis and Mitkas, 2009; Honari et al, 2009). All of these works make important contributions on the research of competing double auction marketplaces, where they undertake empirical analysis in the complex setting for the competing double auction marketplaces. In contrast, we consider a reduced form for this setting to start the theoretical analysis. In Sohn et al (2009), a simple game-theoretic model is proposed to analyse market selection in the CAT context. However, it assumes a game with complete information about the traders' types, in contrast to reality, where traders' types are usually privately known. Finally, in our previous work (Shi et al, 2010a), we have carried out a preliminary analysis on how traders bid and select a marketplace in the context of multiple competing marketplaces and continuous trader types. However, in this work, we did not consider how competing marketplaces set fees to make profits while still maintaining traders.

Another important line of work considering competition between marketplaces is due to McAfee (1993), Burguet and Sakovics (1999) and Gerding et al (2007b), who consider single-sided competition. In particular, they consider competition between sellers who offer similar goods to buyers and can set their own reserve prices to attract buyers in multiple single-sided auctions. The work of McAfee (1993) is based on strong assumptions (e.g. it assumes that any individual seller has no significant impact on buyers' profits), which are only reasonable in the case of infinitely many players. Then Burguet and Sakovics (1999) relax some of these assumptions and show that there always exists an equilibrium for the sellers, but this cannot be a symmetric one in pure strategies. Furthermore, Gerding et al (2007b) show that pure Nash equilibria for the asymmetric seller setting exist. They also extend the above analysis by considering how market fees affect sellers' strategies. However, this model only considers the case with one seller in each marketplace, i.e. single-sided marketplaces. Our setting considering double auction marketplaces with multiple buyers and multiple sellers, is much more complex and the results for the single-sided analysis cannot readily be transferred over.

Furthermore, in addition to the analysis of traders' market selection strategies in the competing marketplace environment, there also exist works regarding bidding strategies across multiple marketplace (e.g. Byde et al, 2002; Anthony and Jennings, 2006; He et al, 2006). However, these works focus on the traders' bidding behaviour across multiple singlesided auctions (such as English Auction, Dutch Auction, etc), and thus cannot be adopted for our double auction setting. In our work, at this stage, we assume that traders adopt a simple bidding strategy (the ray bidding strategy, see Selten and Buchta, 1998; Zhan and Firedman, 2007), although future work is needed to consider the impact of more complex strategies.

The above theoretical works mainly adopt traditional game theory to analyse competing marketplaces. In addition to this, a number of researchers have used evolutionary game theory (EGT) to analyse traders' strategies and market policies (e.g. Phelps et al, 2006, 2010; Vytelingum et al, 2008a). However, their analysis is restricted to isolated double auction marketplaces without considering competition between them. In this paper, we use, for the first time, EGT to analyse traders' market selection strategies as well as the market-

\footnotetext{
3 This is the total profit earned by all traders in the marketplace divided by the maximum possible total profit that could have been earned by all traders.
} 
places' fee strategies in the context of multiple competing marketplaces. This setting is more involved mainly for two reasons. First, we assume incomplete information about traders' types. Second, we model the market as a two-stage game, where in the first stage the marketplaces set their fees, and in the second stage traders choose marketplace conditional on these fees. Although some simple multi-stage games, such as the two-stage ultimatum game (Binmore et al, 1995), have been analysed using EGT, our setting, which considers a game with heterogeneous players and incomplete information, is considerably more complex.

\section{A Game-Theoretic Framework for Competing Marketplaces}

In this section, we introduce the game-theoretic framework which forms the basis of our analysis. In our system, we assume that traders can only choose a single marketplace at a time (called single-homing). We choose this setting because such a trading mechanism results in a highly competitive environment (where marketplaces have to compete fiercely with each other to attract traders) compared to a multi-home trading setting where traders can participate in multiple marketplaces at a time, and we are interested in analysing how both traders and marketplaces behave strategically in such an environment. A trading round in our setting proceeds as follows. First, all marketplaces simultaneously publish their fees. Second, based on observed fees, each trader selects a marketplace according to its market selection strategy. Third, traders submit their offers according to their bidding strategies (in the following, we refer to the offers of buyers as bids and the offers of sellers as asks). Finally, after all traders have submitted their offers, the marketplace matches buyers and sellers and then executes transactions. ${ }^{4}$ In the following, we start by introducing the basic notation of our framework. Then we introduce the notions of marketplaces' fee strategies and traders' market selection strategies respectively. Finally, we provide the definition of equilibrium strategies in the context of our system.

\subsection{Buyers and Sellers}

We consider a set of buyers, $\mathcal{B}=\{1,2, \ldots B\}$, and a set of sellers, $\mathcal{S}=\{1,2, \ldots S\}$. Each buyer is interested in purchasing one item, and each seller has one item for sale. All items are identical. Each buyer and seller has a type, which is denoted as $\theta^{b} \in \Theta^{B}$ and $\theta^{s} \in$ $\Theta^{S}$ respectively, where $\Theta^{B}$ is the set of buyer types and $\Theta^{S}$ is the set of seller types. We assume these sets to be finite. The type of a buyer denotes its limit price, i.e. the highest price it is willing to buy the item for, and the type of a seller denotes its cost price, i.e. the lowest price it is willing to sell the item for. We assume that types of all buyers are independently drawn according to the same probability mass function $f^{b}$ over the finite set of buyer types $\Theta^{B}$, and the types of all sellers are independently drawn according to the same probability mass function $f^{s}$ over the finite set of seller types $\Theta^{S}$. In our framework, the set of types $\Theta^{B}$ and $\Theta^{S}$, and the probability mass functions $f^{b}$ and $f^{s}$ are assumed to be common knowledge. However, the type of each specific trader is not known to the other traders or the marketplaces. In addition, we assume that there is a set of competing marketplaces $\mathcal{M}=\{1,2, \ldots M\}$, that offer places for trade and provide a centralised matching service between the buyers and sellers.

\footnotetext{
4 This means that we consider clearing house double auctions. By adopting this mechanism, and adopting an appropriate matching policy which we will introduce in the following, the marketplaces can match traders in a highly efficient way.
} 


\subsection{Marketplaces and Fees}

Since we consider marketplaces to be commercial enterprises that seek to make a profit, we assume that they charge fees for their service as match makers. To this end, we define a fee structure of a marketplace $m$ to be the tuple $p_{m}=\left(r_{m}, q_{m}\right) \in \mathcal{P}, r_{m} \geq 0$ and $q_{m} \in[0,1]$, where $r_{m}$ is a fixed registration fee charged to a trader when it enters the marketplace, and $q_{m}$ is the so-called profit fee. The latter is a percentage charged on the trader's observed profit, which is defined as the difference between the trader's offer and the transaction price. Furthermore, $\mathcal{P}$ is a finite set of all allowable fee structures. Then the fee structures of all competing marketplaces constitute a fee system $\bar{P}=\left\langle p_{1}, p_{2}, \ldots p_{M}\right\rangle \in \mathcal{P}^{M}$, where $\mathcal{P}^{M}$ is a finite set of all allowable fee systems.

Now we describe how a marketplace will set its fee structure. In this work, we consider a mixed fee strategy, where each fee structure is selected with some probability. A pure strategy can be regarded as a degenerate case of a mixed strategy, where the particular pure strategy is selected with probability 1 and every other strategy with probability 0 . Now, a mixed fee strategy of the marketplace $m$ is defined as $\mu_{m}: \mathcal{P} \rightarrow[0,1]$, which means that the probability that the marketplace $m$ sets fee structure $p_{m}$ is $\mu_{m}\left(p_{m}\right)$, where $\sum_{p_{m} \in \mathcal{P}} \mu_{m}\left(p_{m}\right)=$ 1. We use $\bar{\mu}=\left\langle\mu_{1}(\cdot), \ldots \mu_{M}(\cdot)\right\rangle$ to represent the fee strategy profile of all marketplaces. In addition, we use $\mu_{-m}(\cdot)$ to represent the fee strategy profile of all marketplaces except for marketplace $m$. Then we can rewrite $\bar{\mu}$ as $\bar{\mu}=\left\langle\mu_{m}(\cdot), \mu_{-m}(\cdot)\right\rangle$.

Finally, we use a discriminatory k-pricing policy to determine the transaction price of a matched buyer and seller, which is common in double auction marketplaces. Specifically, the transaction price of a successful interaction in marketplace $m$ is determined by a parameter $k_{m} \in[0,1]$, which sets the transaction price of a matched buyer and seller at the point determined by $k_{m}$ in the interval between their offers. The pricing parameters of all marketplaces constitute the pricing system $\bar{K}=\left\langle k_{1}, k_{2}, \ldots, k_{M}\right\rangle{ }^{5}$

\subsection{Traders' Market Selection Strategies}

After describing the fee strategies of the marketplaces, we now introduce the traders' market selection strategies. We assume that each trader has a mixed market selection strategy, whereby each marketplace is selected with some probability. As we mentioned previously, after all marketplaces publish their fees, traders select a marketplace. Therefore, traders' market selection strategies are conditional on the fee system. Specifically, a mixed market selection strategy of buyer $i$ is defined as a function $\omega_{i}^{b}: \Theta^{B} \times \mathcal{M} \times \mathcal{P}^{M} \rightarrow$ $[0,1]$, where $\omega_{i}^{b}\left(\theta^{b}, m, \bar{P}\right)$ denotes the probability that buyer $i$ with type $\theta^{b} \in \Theta^{B}$ chooses the marketplace $m \in \mathcal{M}$ in the fee system $\bar{P}$, satisfying $\sum_{m \in \mathcal{M}} \omega_{i}^{b}\left(\theta^{b}, m, \bar{P}\right) \leq 1$. Here, $1-\sum_{m \in \mathcal{M}} \omega_{i}^{b}\left(\theta^{b}, m, \bar{P}\right)$ is the probability that buyer $i$ with type $\theta^{b}$ chooses no marketplace. This happens when buyer $i$ finds it has a negative expected profit in each marketplace. We use $\bar{\omega}^{b}(\bar{P})=\left\langle\omega_{1}^{b}(\cdot, \bar{P}), \ldots, \omega_{B}^{b}(\cdot, \bar{P})\right\rangle$ to represent the strategy profile of all buyers in the fee system $\bar{P}$. In addition, we use $\omega_{-i}^{b}(\cdot, \bar{P})$ to represent the strategy profile of all buyers except $i$. Then $\bar{\omega}^{b}(\bar{P})$ can be rewritten as $\bar{\omega}^{b}(\bar{P})=\left\langle\omega_{i}^{b}(\cdot, \bar{P}), \omega_{-i}^{b}(\cdot, \bar{P})\right\rangle$. Similarly, we use $\omega_{j}^{s}: \Theta^{S} \times \mathcal{M} \times \bar{P} \rightarrow[0,1]$ to define the probability of selecting a marketplace of seller $j$ and use $\bar{\omega}^{s}(\bar{P})=\left\langle\omega_{1}^{s}(\cdot, \bar{P}), \ldots, \omega_{S}^{s}(\cdot, \bar{P})\right\rangle$ to represent the strategy profile of all sellers in the fee system $\bar{P}$, and rewrite it as $\bar{\omega}^{s}(\bar{P})=\left\langle\omega_{j}^{s}(\cdot, \bar{P}), \omega_{-j}^{s}(\cdot, \bar{P})\right\rangle$.

\footnotetext{
5 In this work we use a fixed pricing policy and so it does not form part of the strategy of a marketplace, but the framework can be easily extended to include this as part of the strategy as well.
} 
3.4 Definition of Equilibrium Strategies for Selecting a Marketplace and Setting Fees

Before we can analyse how traders select marketplaces and how competing marketplaces set fees (which we discuss in Section 4 and 5 respectively), we first need to specify the expected utility functions for traders and marketplaces, and define an appropriate solution concept in the context of competing marketplaces.

To this end, we first describe a buyer's expected utility equation for a given fee system $\bar{P}$. A seller's expected utility can be given analogously. Given a buyers' strategy profile $\bar{\omega}^{b}(\bar{P})$ and a sellers' strategy profile $\bar{\omega}^{s}(\bar{P})$ in the fee system $\bar{P}$, the expected utility of a buyer $i$ with type $\theta^{b}$ in the fee system $\bar{P}$ is defined by:

$$
\tilde{U}_{i}^{b}\left(\bar{P}, \bar{K}, \bar{\omega}^{b}(\bar{P}), \bar{\omega}^{s}(\bar{P}), \theta^{b}\right)=\sum_{m=1}^{m=M} \omega_{i}^{b}\left(\theta^{b}, m, \bar{P}\right) \times \tilde{U}_{i, m}^{b}\left(\bar{P}, \bar{K}, \bar{\omega}^{b}(\bar{P}), \bar{\omega}^{s}(\bar{P}), \theta^{b}\right)
$$

where $\tilde{U}_{i, m}^{b}\left(\bar{P}, \bar{K}, \bar{\omega}^{b}(\bar{P}), \bar{\omega}^{s}(\bar{P}), \theta^{b}\right)$ is buyer $i$ 's expected utility if it chooses to trade in marketplace $m$.

Furthermore, marketplace $m$ 's expected utility given a fee strategy profile $\bar{\mu}$ and traders' market selection strategy profiles $\bar{\omega}^{b}(\cdot)$ and $\bar{\omega}^{s}(\cdot)$, is as follows:

$$
\tilde{U}_{m}(\bar{\mu})=\sum_{p_{m} \in \mathcal{P}} \mu_{m}\left(p_{m}\right) \times \tilde{U}_{m}\left(p_{m}, \mu_{-m}(\cdot)\right)
$$

where $\tilde{U}_{m}\left(p_{m}, \mu_{-m}(\cdot)\right)$ is marketplace $m$ 's expected utility when its fee structure is $p_{m}$, which is given by:

$$
\tilde{U}_{m}\left(p_{m}, \mu_{-m}(\cdot)\right)=\sum_{\bar{P} \in \mathcal{P} M: p_{m}=\bar{P}(m)} \prod_{l \in \mathcal{M} \backslash\{m\}} \mu_{l}(\bar{P}(l)) \times \tilde{U}_{m}\left(\bar{P}, \bar{\omega}^{b}(\bar{P}), \bar{\omega}^{s}(\bar{P})\right)
$$

where $\tilde{U}_{m}\left(\bar{P}, \bar{\omega}^{b}(\bar{P}), \bar{\omega}^{s}(\bar{P})\right)$ is marketplace $m$ 's expected utility in the fee system $\bar{P}$. Note that both the buyer's expected utility in marketplace $m, \tilde{U}_{i, m}^{b}\left(\bar{P}, \bar{K}, \bar{\omega}^{b}(\bar{P}), \bar{\omega}^{s}(\bar{P}), \theta^{b}\right)$, and marketplace $m$ 's expected utility, $\tilde{U}_{m}\left(\bar{P}, \bar{\omega}^{b}(\bar{P}), \bar{\omega}^{s}(\bar{P})\right)$, depend on the specific bidding strategies adopted by traders and matching policy adopted by marketplace $m$. We will detail them in Section 4.1 and 5.2 respectively where we consider a particular market setting.

After providing general forms for the traders and the marketplaces' expected utilities, now we are ready to define the equilibrium strategies of traders and marketplaces in our system. Since we consider a game with incomplete information about traders' types, the Bayes-Nash equilibrium (BNE) solution concept, in which each player's strategy maximises its expected utility given other players' strategies, is the most appropriate to define this equilibrium behaviour. Here, we define equilibrium strategies of both traders and marketplaces as a whole, since in our system, traders' market selection strategies and marketplaces' fee strategies affect each other. Formally, the mixed Bayes-Nash equilibrium in our setting is defined as:

Definition Given pricing system $\bar{K}$, a fee strategy profile $\bar{\mu}^{*}$ and market selection strategy profiles $\bar{\omega}^{b *}(\cdot)$ and $\bar{\omega}^{s *}(\cdot)$ constitute a mixed Bayes-Nash equilibrium, if

$$
\begin{aligned}
& \forall i \in \mathcal{B}, \forall \theta^{b} \in \Theta^{B}, \forall \bar{P} \in \mathcal{P}^{M}, \forall \omega_{i}^{b}(\cdot, \bar{P}) \in \Delta^{T} \\
& \tilde{U}_{i}^{b}\left(\bar{P}, \bar{K},\left\langle\omega_{i}^{b *}(\cdot, \bar{P}), \omega_{-i}^{b *}(\cdot, \bar{P})\right\rangle, \bar{\omega}^{s *}(\bar{P}), \theta^{b}\right) \geq \tilde{U}_{i}^{b}\left(\bar{P}, \bar{K},\left\langle\omega_{i}^{b}(\cdot, \bar{P}), \omega_{-i}^{b *}(\cdot, \bar{P})\right\rangle, \bar{\omega}^{s *}(\bar{P}), \theta^{b}\right) ; \\
& \text { i.e. each buyer's strategy is a best response to other traders' strategies for each possible } \\
& \text { fee system, }
\end{aligned}
$$


and $\forall j \in \mathcal{S}, \forall \theta^{s} \in \Theta^{S}, \forall \bar{P} \in \mathcal{P}^{M}, \forall \omega_{j}^{s}(\cdot, \bar{P}) \in \Delta^{T}$
$\tilde{U}_{j}^{s}\left(\bar{P}, \bar{K}, \bar{\omega}^{b *}(\bar{P}),\left\langle\omega_{j}^{s *}(\cdot, \bar{P}), \omega_{-j}^{s *}(\cdot, \bar{P})\right\rangle, \theta^{s}\right) \geq \tilde{U}_{j}^{s}\left(\bar{P}, \bar{K}, \bar{\omega}^{b *}(\bar{P}),\left\langle\omega_{j}^{s}(\cdot, \bar{P}), \omega_{-j}^{s *}(\cdot, \bar{P})\right\rangle, \theta^{s}\right) ;$
i.e. each seller's strategy is a best response to other traders' strategies for each possible

fee system,

and $\forall m \in \mathcal{M}, \forall \mu_{m}(\cdot) \in \Delta^{M}$

$\tilde{U}_{m}\left(\left\langle\mu_{m}^{*}(\cdot), \mu_{-m}^{*}(\cdot)\right\rangle\right) \geq \tilde{U}_{m}\left(\left\langle\mu_{m}(\cdot), \mu_{-m}^{*}(\cdot)\right\rangle\right)$

i.e. each marketplace's fee strategy is a best response to other marketplaces' fee strategies,

where $\Delta^{T}$ is the set of all possible (mixed) market selection strategies and $\Delta^{M}$ is the set of all possible (mixed) fee strategies.

Given the equilibrium definition, in what follows, we will analyse both the traders' equilibrium market selection strategies (in Section 4) and the marketplaces' equilibrium fee strategies (in Section 5).

\section{Equilibrium Analysis of the Market Selection Strategies}

Based on the general framework for analysing competing double auction marketplaces, we now use evolutionary game theory to analyse the traders' equilibrium strategies of market selection for a given fee system $\bar{P}=\left\langle p_{1}, \ldots, p_{M}\right\rangle$ (i.e. each marketplace $m \in \mathcal{M}$ sets the fee structure $p_{m}$ with $100 \%$ probability $\left(\mu_{m}\left(p_{m}\right)=1\right)$ ). Before doing this, however, we first need to specify the bidding strategies adopted by traders and the matching policies adopted by the marketplaces. Since the bidding strategy in double auctions is a research area in its own right (well-known strategies include AA-Vytelingum et al, 2008a, GDGjerstad and Dickhaut, 1998, and ZIP-Cliff and Bruten, 1997), and here our focus is on the market selection strategies, we consider a simple bidding strategy for traders. Specifically, we assume that traders use a ray bidding strategy (Turocy, 2001). ${ }^{6}$ Although towards the simple end of the spectrum, this strategy has the property that traders can shade their offers, which means that buyers submit bids less than their types and sellers submit asks higher than their types (in doing so, traders hide their actual types and have the potential to obtain more revenue in the trade). Furthermore, this strategy has a good form of mathematical representation, and thus can be easily incorporated into our mathematical framework. More complex strategies are unsuitable since they are hard to be represented in a mathematical way because they typically rely on ad hoc heuristics and parameter tuning. In more detail, in our system, we assume that the highest and the lowest allowed offer for all traders is $\bar{A}$ and $A$ respectively. Then we use $a^{b}\left(0 \leq a^{b} \leq 1\right)$ to denote the bid factor for buyers, which indicates the degree of buyers shading their offers. Now for a buyer with type $\theta^{b}$, it will shade $\left(1-a^{b}\right) \times\left(\theta^{b}-\underline{A}\right)$, which is from 0 to $\theta^{b}-\underline{A}$. Therefore, it will submit a bid

$$
\eta^{b}\left(\theta^{b}\right)=\theta^{b}-\left(1-a^{b}\right) \times\left(\theta^{b}-\underline{A}\right)=a^{b} \times \theta^{b}+\left(1-a^{b}\right) \times \underline{A}
$$

\footnotetext{
6 The original ray bidding strategy is designed for buyers in single-sided auctions. Specifically, in this bidding strategy, buyers will submit bids as fractions of their types. For example, for the buyer with type $\theta^{b}$, it will submit a bid $\eta^{b}\left(\theta^{b}\right)=a \times \theta^{b}$, where $a$ satisfying $0 \leq a \leq 1$ is the bid factor for buyers, which indicates what factions of values buyers will bid (and also indicates the degree of buyers shading their bids), and therefore $0 \leq \eta^{b}\left(\theta^{b}\right) \leq \theta^{b}$. However, when traders adopt the ray bidding strategy in double auctions, we should note that when buyers submit bids lower than the lowest seller types (or sellers submit asks higher than the highest buyer types), they never make transactions. Therefore, in double auctions, we need to adapt the ray bidding strategy to ensure that traders' offers are within in an appropriate range, in order to guarantee potential transactions. See Equations 4 and 5 for the details.
} 
From this, we can see that, when $a^{b}=1$, buyers bid truthfully, and when $a^{b}=0$, buyers shade at the maximum degree. Similarly, we use $a^{s}\left(0 \leq a^{s} \leq 1\right)$ to indicate the degree of sellers shading their asks. Then a seller with type $\theta^{s}$ will submit an ask:

$$
\eta^{s}\left(\theta^{s}\right)=\theta^{s}+\left(1-a^{s}\right) \times\left(\bar{A}-\theta^{s}\right)=a^{s} \times \theta^{s}+\left(1-a^{s}\right) \times \bar{A}
$$

Similarly, when $a^{s}=1$, sellers will ask truthfully, and when $a^{s}=0$, sellers will shade at the maximum degree. Note that although bidding strategies are known by traders, they will not know what exact offers other traders will submit since they do not know the exact types of their counterparts. For the matching policy, we consider the commonly used equilibrium matching since this aims to maximise traders' profits and thus maximises the allocative efficiency for the marketplace. In detail, this policy will match the buyer with the $v$-th highest bid with the seller with the $v$-th lowest ask if the seller's ask is not greater than the buyer's bid. Given the specific bidding strategy and matching policy, in the following, we will derive traders' expected utilities in this setting, and then analyse traders' equilibrium strategies of market selection for different fee systems. We are interested in calculating the symmetric Bayes-Nash equilibria (BNEs), as is common in game theory for settings with incomplete information, and so we can assume that (in equilibrium) traders with the same type will employ the same strategy. Thus in the following equations, we omit the indexes $i$ and $j$ when referring to specific buyers and sellers.

\subsection{A Trader's Expected Utility}

Before analysing the equilibrium strategies of traders, we first need to calculate their expected utilities, given the ray bidding strategy and equilibrium matching policy. In what follows, we derive the expected utility of a buyer with type $\theta^{b}$ in the fee system $\bar{P}$ when it adopts the ray bidding strategy given the market selection strategy profiles of buyers and sellers, $\bar{\omega}^{b}(\bar{P})$ and $\bar{\omega}^{s}(\bar{P})$. The seller's expected utility is calculated analogously. According to Equation 1, we need to calculate the trader's expected utility in each marketplace $m$. Intuitively, the trader's expected utility in marketplace $m$ not only depends on its own type and bid, but also on other bids and asks appearing in this marketplace. Therefore, we should know the bid and ask distributions for buyers and sellers respectively. Given the ray bidding strategy and the finite set of trader types, we can derive the set of allowable offers for traders. Specifically, we denote the set of allowable bids as $D^{b}=\left\{d^{b} \mid d^{b}=\eta^{b}\left(\theta^{b}\right), \theta^{b} \in \Theta^{B}\right\}$, and denote the set of allowable asks as $D^{s}=\left\{d^{s} \mid d^{s}=\eta^{s}\left(\theta^{s}\right), \theta^{s} \in \Theta^{S}\right\} .{ }^{7}$ Now, given buyers' type distribution function $f^{b}$, market selection strategy profile $\bar{\omega}^{b}(\bar{P})$, we can derive the bid distribution of buyers. For example, the probability of the bid $d^{b}=\eta^{b}\left(\theta^{b}\right)$ submitted by a buyer in marketplace $m$ is:

$$
\phi_{m}^{b}\left(d^{b} \mid \bar{P}\right)=f^{b}\left(\theta^{b}\right) \times \omega^{b}\left(\theta^{b}, m, \bar{P}\right)
$$

Note that this is also the probability that the buyer has type $\theta^{b}$ and chooses marketplace $m$. The probability of the ask $d^{s}$ submitted by a seller in marketplace $m$ is:

$$
\phi_{m}^{s}\left(d^{s} \mid \bar{P}\right)=f^{s}\left(\theta^{s}\right) \times \omega^{s}\left(\theta^{s}, m, \bar{P}\right)
$$

\footnotetext{
7 From Equations 4 and 5, we can see that different types of traders have different offers, and thus $\left|D^{b}\right|=\left|\Theta^{B}\right|$ and $\left|D^{s}\right|=\left|\Theta^{S}\right|$.
} 
Note that the bid and ask distributions are also conditional on the fee system since market selection strategies are conditional on this. Given the bid and ask distributions, we now derive the buyer's expected utility in the following.

First, we introduce four support functions. For buyers, we have:

$$
h_{m}^{b}(d)=\sum_{d^{\prime} \in D^{b}: d^{\prime}>d} \phi_{m}^{b}(d \mid \bar{P})
$$

which denotes the probability that a buyer's bid is strictly higher than $d$ in marketplace $m$;

$$
e_{m}^{b}(d)=\phi_{m}^{b}(d \mid \bar{P})
$$

which denotes the probability that a buyer's bid is equal to $d$ in marketplace $m$; and then $1-h_{m}^{b}(d)-e_{m}^{b}(d)$ is the probability that the buyer's bid is strictly less than $d$ in marketplace $m$ or the buyer does not choose marketplace $m$. Similarly, for sellers, we have:

$$
h_{m}^{s}(d)=\sum_{d^{\prime} \in D^{s}: d^{\prime}<d} \phi_{m}^{s}(d \mid \bar{P})
$$

which denotes the probability that a seller's ask is strictly less than $d$ in marketplace $m$;

$$
e_{m}^{s}(d)=\phi_{m}^{s}(d \mid \bar{P})
$$

which denotes the probability that a seller's ask is equal to $d$ in marketplace $m$; and then $1-h_{m}^{s}(d)-e_{m}^{s}(d)$ is the probability that the seller's ask is strictly higher than $d$ in marketplace $m$ or the seller does not choose marketplace $m$.

Now we begin to derive the buyer's expected utility in marketplace $m$ with bid $d^{b}\left(d^{b}=\right.$ $\eta^{b}\left(\theta^{b}\right)$ ). Since we consider equilibrium matching policy to match traders, we need to know the position of the buyer's bid among all bids in marketplace $m$, which depends on other buyers' bids. Specifically, we use

$$
\rho^{b}\left(x, y, d^{b}\right)=\left(\begin{array}{c}
B-1 \\
x, y
\end{array}\right) \times\left(h_{m}^{b}\left(d^{b}\right)\right)^{x} \times\left(e_{m}^{b}\left(d^{b}\right)\right)^{y} \times\left(1-h_{m}^{b}\left(d^{b}\right)-e_{m}^{b}\left(d^{b}\right)\right)^{B-1-x-y}
$$

to calculate the probability that in marketplace $m$, there are exactly $x$ buyers' bids strictly higher than $d^{b}$, exactly $y$ buyers' bids tying with $d^{b}$ (excluding the buyer for which we are calculating the expected utility), and exactly $B-1-x-y$ buyers' bids strictly less than $d^{b}$ or not choosing marketplace $m$. In this situation, the buyer's position in marketplace $m, v$, can be anywhere between $x+1$ and $x+y+1$. We use a tie-breaking rule where each of these possible positions occurs with equal probability $1 /(y+1)$. We denote the buyer's expected utility given its position $v$ as $\tilde{U}_{m}^{b}\left(\bar{P}, \bar{K}, \bar{\omega}^{b}(\bar{P}), \bar{\omega}^{s}(\bar{P}), \theta^{b}, v\right)$. Now the expected utility of the buyer in marketplace $m$ is given by:

$$
\tilde{U}_{m}^{b}\left(\bar{P}, \bar{K}, \bar{\omega}^{b}(\bar{P}), \bar{\omega}^{s}(\bar{P}), \theta^{b}\right)=\sum_{x=0}^{B-1} \sum_{y=0}^{B-1-x} \rho^{b}\left(x, y, d^{b}\right) \times \frac{1}{y+1} \times \sum_{v=x+1}^{x+y+1} \tilde{U}_{m}^{b}\left(\bar{P}, \bar{K}, \bar{\omega}^{b}(\bar{P}), \bar{\omega}^{s}(\bar{P}), \theta^{b}, v\right)-r_{m}
$$

where $r_{m}$ is the registration fee charged to the buyer when it enters the marketplace $m$. We now calculate the buyer's expected utility when its bid is $v$-th highest among all bids in marketplace $m$, which is given by:

$$
\tilde{U}_{m}^{b}\left(\bar{P}, \bar{K}, \bar{\omega}^{b}(\bar{P}), \bar{\omega}^{s}(\bar{P}), \theta^{b}, v\right)=\sum_{d^{s} \in D^{s}: d^{s} \leq d^{b}} \tilde{U}_{m}^{b}\left(\bar{P}, \bar{K}, \bar{\omega}^{b}(\bar{P}), \bar{\omega}^{s}(\bar{P}), \theta^{b}, v, d^{s}\right)
$$


where $\tilde{U}_{m}^{b}\left(\bar{P}, \bar{K}, \bar{\omega}^{b}(\bar{P}), \bar{\omega}^{s}(\bar{P}), \theta^{b}, v, d^{s}\right)$ is the buyer's expected utility when it attempts to be matched with the ask $d^{s}$, which is given by:

$$
\tilde{U}_{m}^{b}\left(\bar{P}, \bar{K}, \bar{\omega}^{b}(\bar{P}), \bar{\omega}^{s}(\bar{P}), \theta^{b}, v, d^{s}\right)=\sum_{r=0}^{v-1} \sum_{t=v-r}^{S-r} \rho^{s}\left(r, t, d^{s}\right) \times \tilde{U}_{m \mid d^{s}}^{b}\left(\bar{P}, \bar{K}, \bar{\omega}^{b}(\bar{P}), \bar{\omega}^{s}(\bar{P}), \theta^{b}, v\right)
$$

where

$$
\rho^{s}\left(r, t, d^{s}\right)=\left(\begin{array}{c}
S \\
r, t
\end{array}\right) \times\left(h_{m}^{s}\left(d^{s}\right)\right)^{r} \times\left(e_{m}^{s}\left(d^{s}\right)\right)^{t} \times\left(1-h_{m}^{s}\left(d^{s}\right)-e_{m}^{s}\left(d^{s}\right)\right)^{S-r-t}
$$

is the probability that there are exactly $r$ asks strictly less than $d^{s}$ and exactly $t$ asks equal to $d^{s}$ (including the ask itself). Note that $t$ should be at least equal to $v-r$ in Equation 15, and thus $\sum_{r=0}^{v-1} \sum_{t=v-r}^{S-r} \rho^{s}\left(r, t, d^{s}\right)$ actually gives the overall probability that the bid $d^{b}$ is matched with the ask $d^{s}$. Finally, $\tilde{U}_{m \mid d^{s}}^{b}\left(\bar{P}, \bar{K}, \bar{\omega}^{b}(\bar{P}), \bar{\omega}^{s}(\bar{P}), \theta^{b}, v\right)$ is the buyer's expected utility when it is matched with the ask $d^{s}$. This is given by:

$$
\tilde{U}_{m \mid d^{s}}^{b}\left(\bar{P}, \bar{K}, \bar{\omega}^{b}(\bar{P}), \bar{\omega}^{s}(\bar{P}), \theta^{b}, v\right)=\theta^{b}-d^{b}+\left(d^{b}-\mathrm{TP}\right) \times\left(1-q_{m}\right)
$$

where TP $=d^{s} \times k_{m}+d^{b} \times\left(1-k_{m}\right)$ is the transaction price, and $d^{b}-\mathrm{TP}$ is the buyer's observed profit, and $q_{m}$ is the profit fee charged to traders.

The above equation gives the expected utility of the buyer in a particular marketplace. Therefore, a buyer's expected utility over all marketplaces in the fee system $\bar{P}$ is:

$$
\tilde{U}^{b}\left(\bar{P}, \bar{K}, \bar{\omega}^{b}(\bar{P}), \bar{\omega}^{s}(\bar{P}), \theta^{b}\right)=\sum_{m=1}^{M} \omega^{b}\left(\theta^{b}, m, \bar{P}\right) \times \tilde{U}_{m}^{b}\left(\bar{P}, \bar{K}, \bar{\omega}^{b}(\bar{P}), \bar{\omega}^{s}(\bar{P}), \theta^{b}\right)
$$

After deriving traders' expected utilities, in the next section, we will use EGT to analyse the equilibrium market selection strategies of traders.

\subsection{An Evolutionary Analysis of Market Selection Strategies}

We now use the equations from the previous section together with EGT to analyse the traders' equilibrium market selection strategies for a given fee system $\bar{P}=\left\langle p_{1}, \ldots, p_{M}\right\rangle$. Specifically, we will use replicator dynamics to computationally determine the equilibrium strategies. Replicator dynamics is used in evolutionary game theory (EGT) to control the learning process, in which players gradually adjust their strategies over time in response to the repeated observation of their opponents' strategies. In the following section, we will describe the replicator dynamics equations, which capture the dynamics of how traders evolving to equilibrium market selection strategies, and then give the evolutionary analysis in detail. Before doing these, we first detail the assumptions used in our analysis.

Firstly, since we focus on market selection strategies, we assume that traders adopt a fixed bid factor for the ray bidding strategy during the analysis. ${ }^{8}$ Furthermore, we assume that there are only two types of buyers and two types of sellers: ${ }^{9}$ rich and poor, which are denoted by $\theta_{2}^{b}$ and $\theta_{1}^{b}$ respectively for buyers, and $\theta_{1}^{s}$ and $\theta_{2}^{s}$ for sellers, i.e. $\Theta^{B}=\left\{\theta_{1}^{b}, \theta_{2}^{b}\right\}$

\footnotetext{
8 In the future, we would like to analyse how traders evolve to the equilibrium ray bidding strategies.

9 Although we only consider two types of traders, our general framework indeed allows the analysis of more types of traders. However, introducing more types will significantly increase the number of possible starting points as well as the number of replicator dynamics equations, making the computation intractable. Therefore, here we consider 2 types of traders.
} 
and $\Theta^{S}=\left\{\theta_{1}^{s}, \theta_{2}^{s}\right\}$. A rich buyer is defined as having a higher limit price than a poor buyer, i.e. $\theta_{2}^{b}>\theta_{1}^{b}$, and a rich seller is defined as having a lower cost price than a poor seller, i.e. $\theta_{1}^{s}<\theta_{2}^{s}$. Trader types are independently drawn with an equal probability (i.e. $f^{b}\left(\theta_{1}^{b}\right)=$ $f^{b}\left(\theta_{2}^{b}\right)=0.5$ and $f^{s}\left(\theta_{1}^{s}\right)=f^{s}\left(\theta_{2}^{s}\right)=0.5$ ). Furthermore, in this paper, we assume that rich buyers and rich sellers have the same evolutionary behaviour and poor buyers and poor sellers have the same evolutionary behaviour. ${ }^{10}$ Therefore, during the evolutionary process, rich(poor) buyers should have the same expected utility as rich(poor) sellers. In order to reasonably make this assumption, rich(poor) buyers and rich(poor) sellers should be treated equally by marketplaces. Thus we assume pricing parameter $k_{m}=0.5$, i.e. the transaction price is set in the middle of offers of the matched buyers and sellers, which means that the marketplaces have no bias in favor of buyers or sellers when allocating trading profits. Furthermore, we should assume that the profits of buyers and sellers are symmetric and therefore, as an example, we let $\theta_{1}^{b}=4, \theta_{2}^{b}=6, \theta_{1}^{s}=0$ and $\theta_{2}^{s}=2$, and all traders adopt the same bid factor in the ray bidding strategy. Furthermore, in our system, we assume that the highest allowed offer is 6 (i.e. $\bar{A}=6$ ) and the lowest allowed offer is 0 (i.e. $A=0$ ). In addition, we assume that there are equal numbers of buyers and sellers. Specifically, we assume that there are 5 buyers and 5 sellers. ${ }^{11}$

\subsubsection{Replicator Dynamics}

The replicator dynamics equations specify the dynamic adjustment of the probability with which pure strategy should be played (for example, in Phelps et al, 2010, replicator dynamics are used to show how traders bid in a double auction marketplace). Since we assume that rich buyers and rich sellers have the same learning behaviour, and poor buyers and poor sellers have the same learning behaviour, in this work, we have 2 populations (i.e. rich traders and poor traders). We first introduce the 2-population replicator dynamics equations which show the dynamic changes of traders' market selection strategies with respect to time $t$. Note that here a time step corresponds to an evolutionary step.

$$
\begin{aligned}
\dot{\omega}^{b}\left(\theta_{1}^{b}, m, \bar{P}\right)=\dot{\omega}^{s}\left(\theta_{2}^{s}, m, \bar{P}\right) & =\frac{d \omega^{b}\left(\theta_{1}^{b}, m, \bar{P}\right)}{d t} \\
& =\left(\tilde{U}_{m}^{b}\left(\bar{P}, \bar{K}, \bar{\omega}^{b}(\bar{P}), \bar{\omega}^{s}(\bar{P}), \theta_{1}^{b}\right)-\tilde{U}^{b}\left(\bar{P}, \bar{K}, \bar{\omega}^{b}(\bar{P}), \bar{\omega}^{s}(\bar{P}), \theta_{1}^{b}\right)\right) \times \omega^{b}\left(\theta_{1}^{b}, m, \bar{P} 19\right) \\
\dot{\omega}^{b}\left(\theta_{2}^{b}, m, \bar{P}\right)=\dot{\omega}^{s}\left(\theta_{1}^{s}, m, \bar{P}\right) & =\frac{d \omega^{b}\left(\theta_{2}^{b}, m, \bar{P}\right)}{d t} \\
& =\left(\tilde{U}_{m}^{b}\left(\bar{P}, \bar{K}, \bar{\omega}^{b}(\bar{P}), \bar{\omega}^{s}(\bar{P}), \theta_{2}^{b}\right)-\tilde{U}^{b}\left(\bar{P}, \bar{K}, \bar{\omega}^{b}(\bar{P}), \bar{\omega}^{s}(\bar{P}), \theta_{2}^{b}\right)\right) \times \omega^{b}\left(\theta_{2}^{b}, m, \bar{P} \not 0\right)
\end{aligned}
$$

As an example, $\dot{\omega}^{b}\left(\theta_{1}^{b}, m, \bar{P}\right)$ describes how the poor buyer with type $\theta_{1}^{b}$ changes its probability of choosing marketplace $m$ in the fee system $\bar{P}$. Here, $\tilde{U}_{m}^{b}\left(\bar{P}, \bar{K}, \bar{\omega}^{b}(\bar{P}), \bar{\omega}^{s}(\bar{P}), \theta_{1}^{b}\right)$ is the poor buyer's expected utility when it chooses marketplace $m$, and $\tilde{U}^{b}\left(\bar{P}, \bar{K}, \bar{\omega}^{b}(\bar{P}), \bar{\omega}^{s}(\bar{P})\right.$, $\theta_{1}^{b}$ ) is the poor buyer's overall expected utility (see Subsection 4.1). In order to get the dynamics of the strategies, we need to calculate trajectories, which indicate how the mixed strategies evolve. In more detail, initially, a mixed strategy is chosen as a starting point (in our results, we experiment with a large number of such points). The dynamics are then calculated according to the above replicator equations. According to the dynamic changes of

\footnotetext{
10 In Shi et al (2010b), we also ran experiments considering that rich(poor) buyers and rich(poor) sellers have different behaviour. However, we found that results are broadly similar, and so we do not present them in this paper.

11 In the experimental analysis, we also ran experiments with more buyers and more sellers. However, the results with higher number of traders are similar and so are not included here.
} 
traders' strategies, their current mixed strategies can be calculated. Such calculations are repeated until $\dot{\omega}^{b}(\cdot)$ becomes zero, at which point the equilibrium for the replicator dynamics is reached (which is also called the rest point for the replicator dynamics). If the equilibrium for the replicator dynamics is asymptotically stable, then this equilibrium is a Nash equilibrium. ${ }^{12}$ When considering traders evolving from all possible starting points, we get several regions. The region from which all trajectories converge to a particular equilibrium is called the basin of attraction of this equilibrium. The basin is very useful since, given the assumption that each starting point is selected by traders with an equal probability, its size can be used as an indicator of the probability of traders converging to that equilibrium (Tuyls and Parsons, 2007).

\subsubsection{Experimental Results}

After providing replicator dynamics equations, we now analyse the equilibrium market selection strategies for traders. We first consider 2 competing marketplaces, and in Section 4.2.4, we will extend the analysis to more marketplaces. Note that, in our framework, traders' market selection strategies are conditional on the fee system and we consider both registration and profit fees. Therefore, in the following, we analyse the equilibrium market selection strategies in different cases where marketplaces charge each possible type of fee.

\section{Both marketplaces only charging profit fees:}

We first analyse the equilibrium market selection strategies when both marketplaces only charge profit fees. In this case, traders will always choose one of the marketplaces since traders have non-negative profits when participating. As an illustrative example, we assume that marketplace 1 charges $20 \%$ profit fee and marketplace 2 charges $30 \%$ profit fee. For the bid factor of traders' ray bidding strategies, we first assume that $a^{b}=a^{s}=1$, i.e. traders bid truthfully. Then the dynamic results for different starting points are shown in Figure 1(a), where the $x$-axis is the rich buyer(seller)'s probability of choosing market 1 , and the $y$-axis is the poor buyer(seller)'s probability of choosing marketplace 1 . We find that all traders either converge to BNE 1 (i.e. marketplace 1) or BNE 2 (i.e. marketplace 2) depending on the initial conditions. The figure also shows that the basin of attraction to BNE 1 is bigger. This means that, (assuming each starting point has an equal probability) since marketplace 1 charges less, traders have a higher probability of converging to marketplace 1.

For the same setting, we now analyse how equilibrium market selection strategies are changed when traders can shade their offers. Specifically, we assume that $a^{b}=a^{s}=0.8$. Now the evolutionary results are shown in Figure 1(b), from which we can see that traders still converge to marketplace 1 or marketplace 2 in equilibrium. However, comparing Figure 1(b) with Figure 1(a), we can see that traders have a slightly higher probability of choosing marketplace 2 when traders can shade their offers than when traders bid truthfully. This is because, when traders shade their offers, their absolute payments incurred by profit fees are reduced since the observed profits are reduced, and therefore traders are less sensitive to the difference of the two marketplaces' profit fees. Therefore, in Figure 1(b), traders only have a slightly higher probability of choosing marketplace 1 than that of choosing marketplace 2. From Figures 1(a) and 1(b), we can furthermore see that two competing marketplaces never co-exist in equilibrium. Similar results are obtained with different combinations of profits

\footnotetext{
12 An equilibrium in replicator dynamics is asymptotically stable if: a) any solution path of the replicator dynamics remains arbitrarily close to the equilibrium if it starts sufficiently close to it; b) any solution path of the replicator dynamics converges to the equilibrium if it starts close enough to it (Tuyls and Parsons, 2007). In Redondo (2003), it is proved that if an equilibrium of the replicator dynamics is asymptotically stable, then it is a Nash equilibrium.
} 


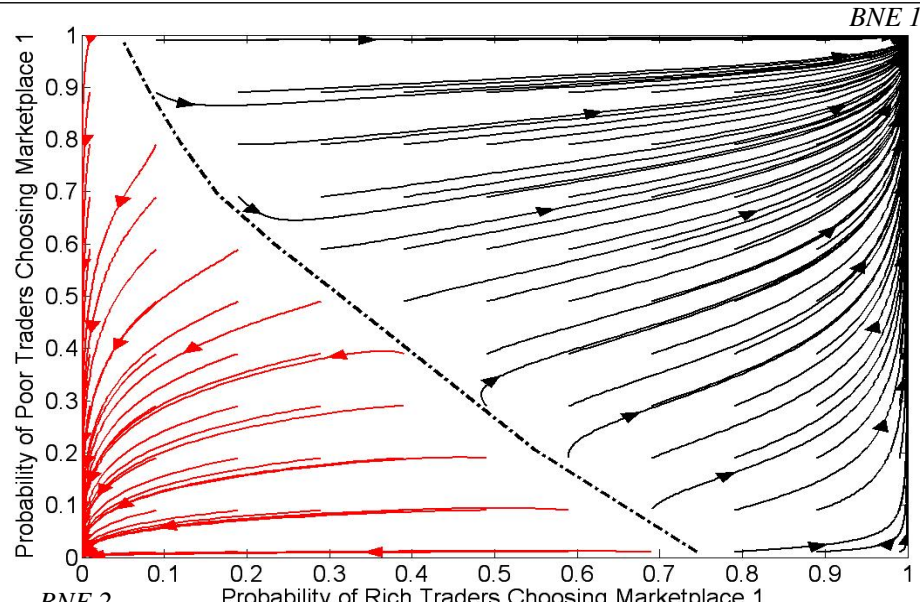

(a) Traders bid truthfully, i.e. $a^{b}=a^{s}=1$.

$B N E 1$

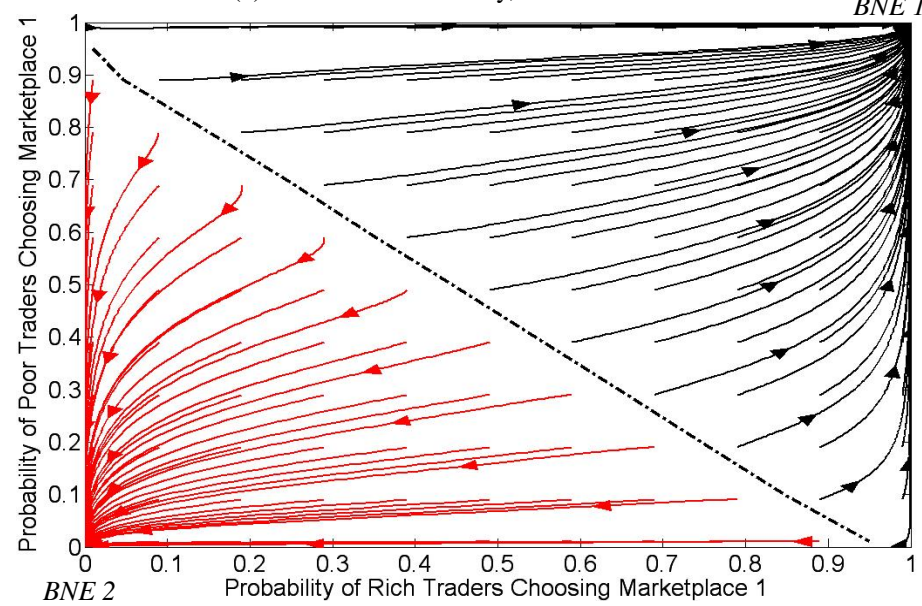

(b) Traders shade their offers with $a^{b}=a^{s}=0.8$.

Fig. 1: Evolutionary process of market selection strategies when marketplace 1 charges $20 \%$ profit fee and 2 charges $30 \%$ profit fee. The dotted line denotes the boundary between the basins of attractions.

fees charged by marketplaces (see the experimental results in Figures 16 and 17 in Appendix A, in which we use the different gray levels to indicate the probability of traders choosing each marketplace in equilibrium). This shows that, in our framework, the positive network effect (where traders prefer marketplaces with a larger number of traders on the other side) has a larger impact than the negative network effect (where traders prefer marketplaces with fewer other traders on the same side), which will cause traders to converge to one marketplace in equilibrium.

\section{Both marketplaces only charging registration fees:}

We now extend the above analysis to the case where both competing marketplaces only charge registration fees. In this case, we should note that traders may not choose any marketplaces since their expected profits may be negative. Therefore, now there are three pure 
market selection strategies for traders, i.e. choosing marketplace 1, choosing marketplace 2, and choosing no marketplace. As a result, we cannot show the evolutionary results from all starting points in a 2-dimension graph, and instead, in the following, in this section we show the evolutionary process from a single starting point as an example. However, in Appendix A, we indeed show the results from other starting points and other possible registration fees. We first assume that traders bid truthfully, i.e. $a^{b}=a^{s}=1$. Figure 2(a) shows the evolutionary process when marketplace 1 charges a 0.5 registration fee, and marketplace 2 charges a 0.8 registration fee. Recall that time corresponds to evolutionary steps. From this we can see that all traders converge to marketplace 1, which is the cheaper one. However, when marketplace 1 charges a 1.5 registration fee, and marketplace 2 charges a 1.8 registration fee, from Figure 2(b), we can see that rich traders will converge to marketplace 1, and poor traders will not choose any marketplace because high registration fees cause negative profits for them. We find similar results when running experiments from other starting points and different combinations of registration fees. That is, traders always choose either the same marketplace or no marketplace, and never distribute in two competing marketplaces. These results for different settings are shown in Figures 18 and 19 in Appendix A. Furthermore, we run experiments by considering that traders shade their offers. We find that the bid factors do not affect the traders' market selection strategies significantly since the absolute payments incurred from registration fees are not affected by shading. Therefore, the conclusions are similar to the case where traders bid truthfully.

Marketplace 1 charging a profit fee and marketplace 2 charging a registration fee: In reality, it also happens that different marketplaces charge different types of fees. We now analyse traders' equilibrium market selection strategies in this situation. Specifically, we assume that marketplace 1 charges a profit fee, and marketplace 2 charges a registration fee. In this situation, traders will always choose one of the marketplaces (since at least marketplace 2 can guarantee non-negative profits for them). We find that for some fee systems, ${ }^{13}$ when evolving from some starting points, traders may converge to different marketplaces in equilibrium. As an illustrative example, we assume that marketplace 1 charges $70 \%$ profit fee and marketplace 2 charges 0.8 registration fee, and traders bid truthfully. The results are shown in Figure 3(a). We find that, in this case, in addition to BNE 1 and BNE 2, there exists a third equilibrium (BNE 3), where rich traders converge to marketplace 1 which charges a registration fee, and poor traders converge to marketplace 2 which charges a profit fee. At this moment, two competing marketplaces co-exist. In contrast to Ellison et al (2004), where co-existence of competing marketplaces is caused by negative network effects, here the co-existence is caused by the strong differentiation of competing marketplaces by setting different types of fees. In more detail, rich traders prefer the marketplace charging a lump sum fee since this fee is smaller than the absolute payment incurred from charging profit fees on a large observed profit. However, poor traders prefer the marketplace which charges a profit fee, since this can guarantee non-negative profits for them, and a high registration fee may lead to negative profits. ${ }^{14}$ Similarly, the same phenomenon is observed when traders shade their offers. As an example, Figure 3(b) shows the evolutionary results

\footnotetext{
13 Specifically, in this experimental setting, the profit fee of marketplace 1 is higher than $10 \%$, and the registration fee of marketplace 2 is higher than 0.4 .

14 For example, for the starting points that rich traders choose marketplace 1 and 2 with probability 0.3 and 0.7 respectively, and poor traders choose marketplace 1 and 2 with probability 0.8 and 0.2 respectively, when marketplace 1 charges $50 \%$ profit fee and marketplace 2 charges 0.8 registration fee, the expected payment for rich traders in marketplace 1 is 1.17 , which is higher than that in marketplace 2 , which is 0.8 . Therefore, rich traders prefer marketplace 2 . In contrast, the expected payment of poor traders in marketplace 1 is 0.45 , which is smaller than that in marketplace 2 , which is 0.8 . Then poor traders prefer marketplace 1.
} 


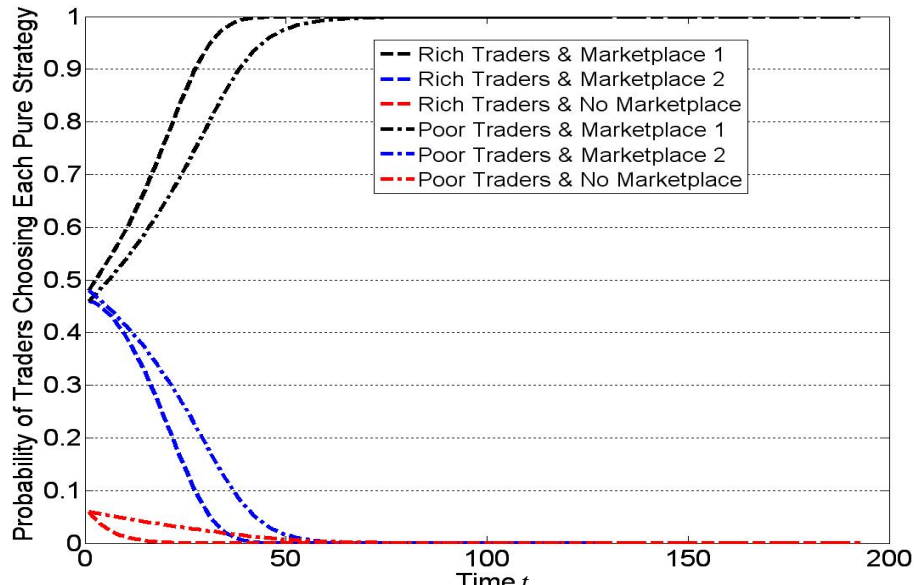

(a) Marketplace 1 charges a 0.5 registration fee, and marketplace 2 charges a 0.8 registration fee.

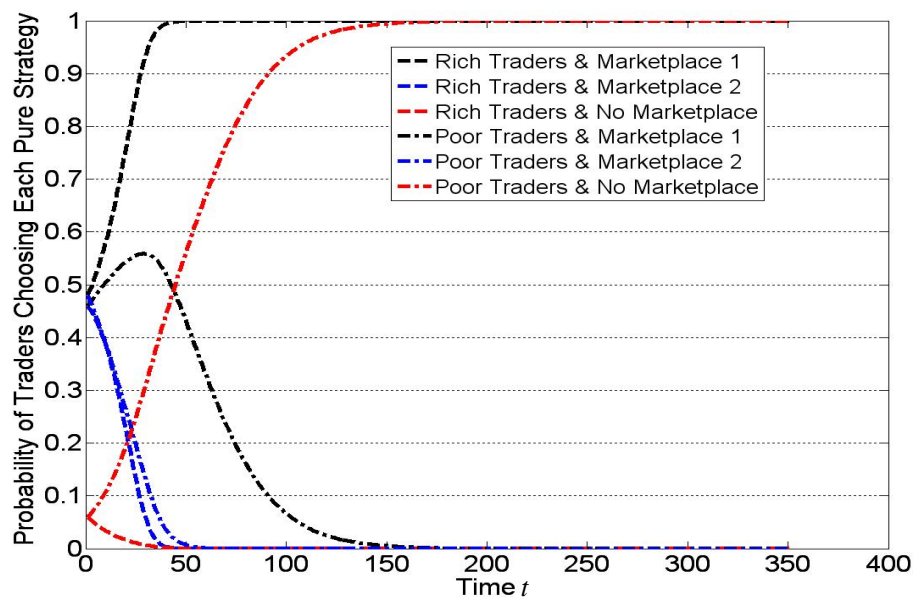

(b) Marketplace 1 charges a 1.5 registration fee, and marketplace 2 charges a 1.8 registration fee.

Fig. 2: Evolutionary process of market selection strategies when both competing marketplaces charge registration fees, and initially rich traders choose marketplace 1, 2 and no marketplace with probability $0.48,0.46$ and 0.06 respectively, and poor traders choose marketplace 1,2 and no marketplace with probability $0.46,0.48$ and 0.06 respectively. Traders bid truthfully, i.e. $a^{b}=a^{s}=1$.

when $a^{b}=a^{s}=0.8$. We can see that when evolving from some starting points, rich traders will converge to marketplace 2 charging a registration fee, and poor traders will converge to marketplace 1 charging a profit fee, i.e. two competing marketplaces can co-exist. However, since traders can shade offers, and thus the payments incurred by profit fees are reduced, comparing Figure 3(a) with Figure 3(b), we can see that traders have a higher probability of choosing the marketplace charging a profit fee (i.e. BNE 1). Thus, as traders shade, the basin of attraction of marketplace 1 becomes larger. Experimental results using different registration and profit fees are shown in Figures 20, 21, 22 and 23 in Appendix A. In all of these cases, we still find the same conclusions, where different types of traders may converge to 

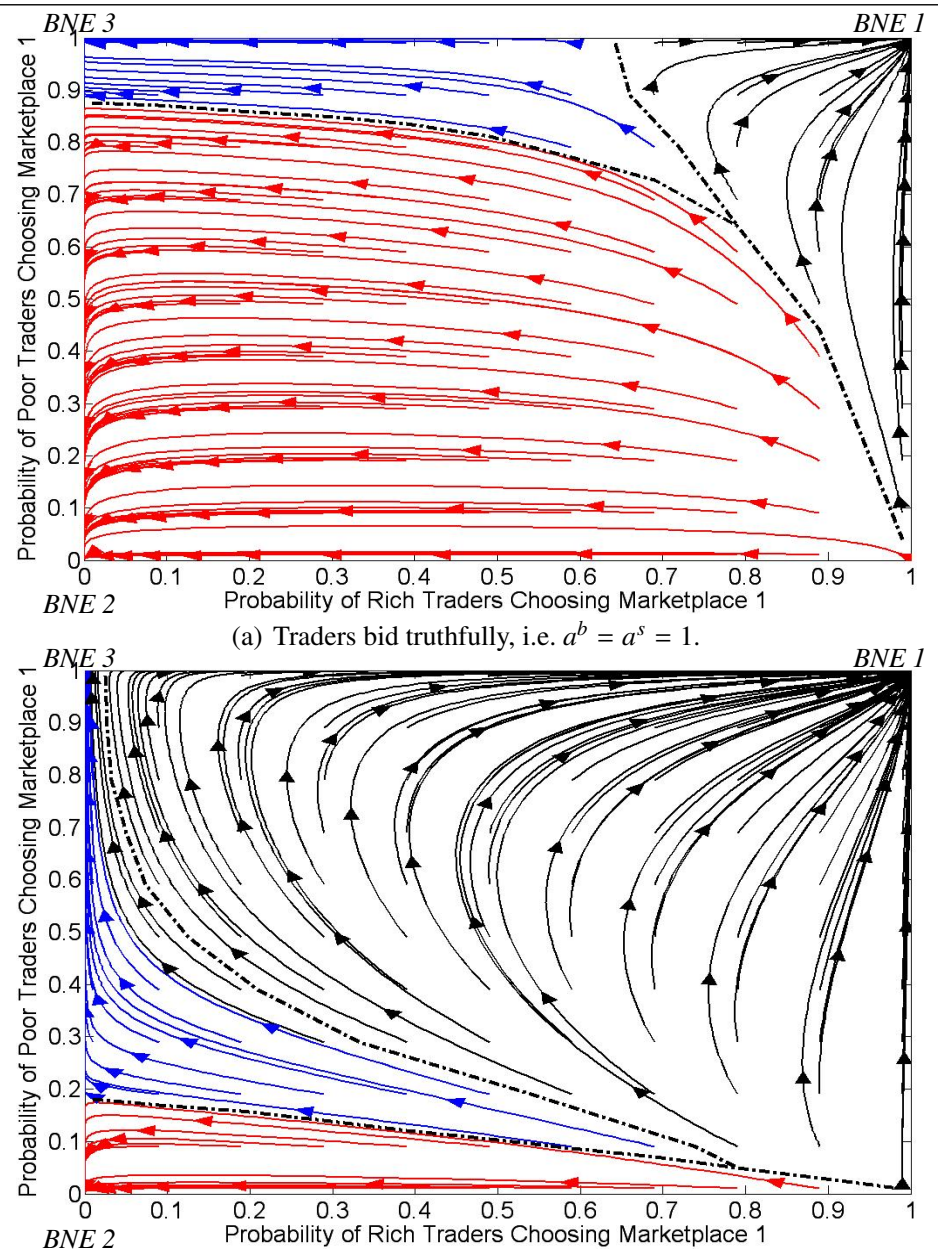

(b) Traders shade their offers with $a^{b}=a^{s}=0.8$

Fig. 3: Evolutionary process of market selection strategies when marketplace 1 charges $70 \%$ profit fee and marketplace 2 charges 0.8 registration fee. The dotted line denotes the boundary between the basins of attractions.

different marketplaces in equilibrium, and when traders shade, they prefer to choose marketplace 1 charging a profit fee.

Both marketplaces charging combinations of registration and profit fees:

We now extend the above analysis to the more general case that both competing marketplaces can charge a combination of registration and profit fees. In this case, we find that traders may converge to the same marketplace, or may not choose any marketplace, or different types of traders may converge to different marketplaces. For example, we assume that traders bid truthfully, and marketplace 1 charges 0.1 registration fee and $50 \%$ profit fee, and marketplace 2 charges 0.8 registration fee and $10 \%$ profit fee. The evolutionary process of traders evolving from a specific starting point is shown in Figure 4. From this figure, we can see that rich traders converge to marketplace 2 which charges a higher registration fee and a 


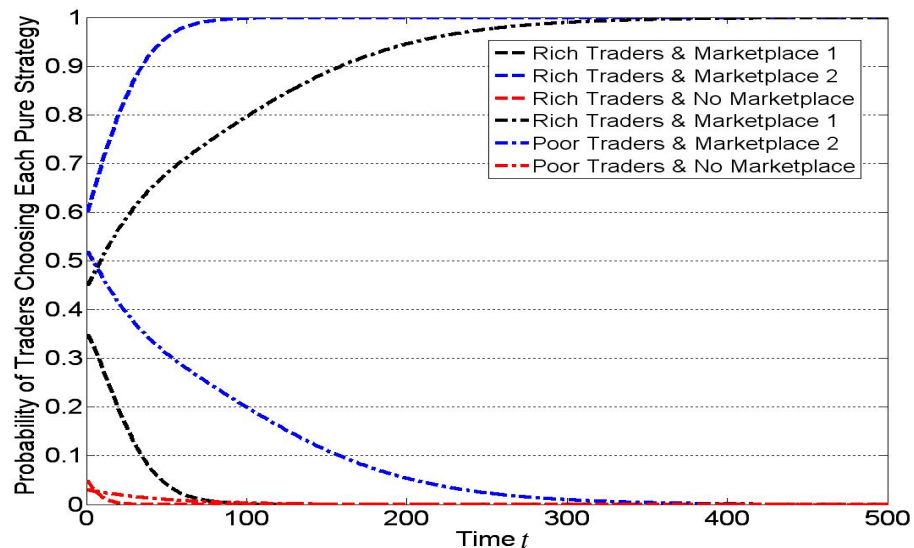

Fig. 4: Evolutionary process of market selection strategies when marketplace 1 charges 0.1 registration fee and 50\% profit fee, marketplace 2 charges 0.8 registration fee and $10 \%$ profit fee, and initially rich traders choose marketplace 1,2 and no marketplace with probability $0.35,0.6$ and 0.05 respectively, and poor traders choose marketplace 1,2 and no marketplace with probability $0.45,0.52$ and 0.03 respectively. Traders bid truthfully, i.e. $a^{b}=a^{s}=1$.

lower profit fee, and poor traders converge to marketplace 1 which charges a lower registration fee and a higher profit fee. When exploring other fee systems (see Figures 24 and 25), we still can find the similar conclusions, where depending on the initial starting points and fees, traders may converge to the same marketplace (or choose no marketplace), or different types of traders may converge to different marketplaces. Furthermore, when traders can shade their offers, we still find the similar conclusions.

\subsubsection{Lock-in Region}

In the above analysis, we can see that, due to network effects, when evolving from certain starting points, traders may converge to the expensive marketplace in equilibrium (for example, in Figure 1(a), we can see that traders may converge to marketplace 2 in equilibrium). This is interesting since it means that the marketplace can charge higher fees to make more profits but still keep traders (even if the size of the basin of attraction is smaller when fees are relatively higher). In the following, we analyse this in detail. In doing so, we consider profit fees as an example. Since we consider a finite set of fees in our framework, we discretize the continuous profit fees of the marketplaces. Specifically, we discretize the continuous profit fee from 0 and 1 with a step size of 0.1 . Clearly, the traders' evolution of their market selection strategies depends on two factors: the starting point and the fees charged to them. We now choose a starting point where rich traders choose marketplace 1 and 2 with probability 0.8 and 0.2 respectively, and poor traders choose marketplace 1 and 2 with probability 0.7 and 0.3 respectively (i.e. traders initially have a higher probability of choosing marketplace 1). We also assume that traders bid truthfully (in the following, we will analyse this when traders can shade their offers). Figure 5 then shows the results after we evolve the traders' market selection strategies in the competing marketplaces with different profit fees. The shaded area is what we call the "lock-in region", which shows when profit fees of marketplace 1 and 2 are within this area, traders converge to marketplace 1 , even though marketplace 1 charges a higher profit fee than marketplace 2 . This result shows that, the expensive marketplace can make more profits while still maintaining traders. Note 


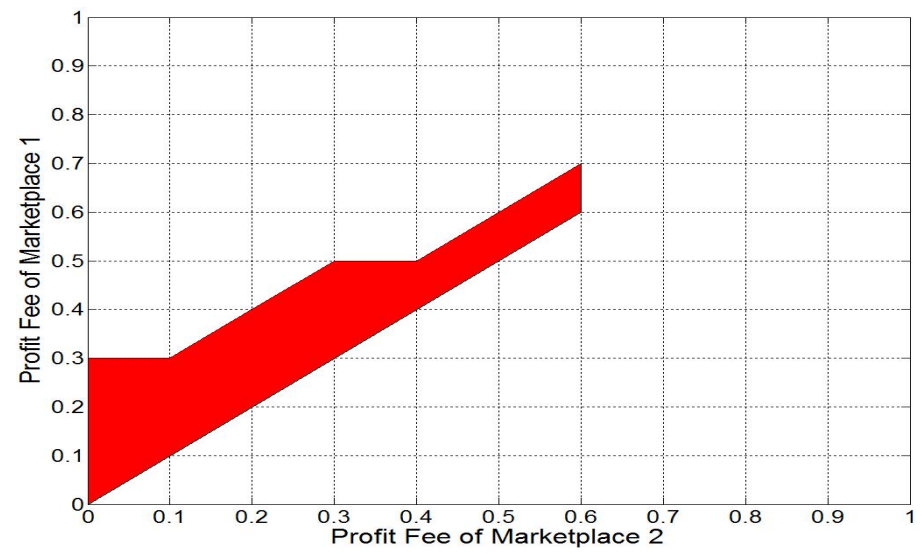

Fig. 5: Lock-in region when rich traders choose marketplace 1 and 2 with probability 0.8 and 0.2 respectively, and poor traders choose marketplace 1 and 2 with probability 0.7 and 0.3 respectively. Traders bid truthfully, i.e. $a^{b}=a^{s}=1$.

that, when the profit fee of marketplace 2 is higher than $60 \%$, marketplace 1 can no longer maintain traders if its profit fee is higher than marketplace 2, i.e. the lock-in region disappears. We find similar lock-in regions when both competing marketplaces charge registration fees, charge different types of fees, or charge combinations of profit and registration fees. Now we can see that it is possible for traders to converge to the expensive marketplace if currently traders have higher probabilities of choosing this marketplace. This result gives useful insights into a strategy for setting fees in competing marketplaces. Specifically, firstly, a marketplace should lower its fees to attract or maintain traders. After obtaining an advantageous position, the marketplace should then increase its fees higher than its opponents, while still keeping its traders since traders still have higher expected utilities in the expensive marketplace. This so-called bait-and-switch strategy has been adopted by a number of entrants in the CAT competition (Niu et al, 2008), where initially they charge lower and even no fees to attract traders, and once they have built up a larger market share, they will charge fees to make profits, but still can maintain market share at a good level. While such a strategy is quite intuitive and common in many marketplaces, our analysis provides a more formal justification for it. Furthermore, we can use the strategy as an indication of the level at which the fees should be set.

We now consider how the bid factor affects the size of the lock-in region when both marketplaces only charge profit fees. First, we calculate the size of the lock-in region as the sum of the differences of two marketplaces' discretized profit fees in the lock-in region. For example, the size of the lock-in region in Figure 5 is 1.2. ${ }^{15}$ We consider the same starting point as the above analysis. For the ray bidding strategy, we assume that the bid factor is in the range of 0.75 to $1 .{ }^{16}$ The relationship between the bid factor and the size of the lock-in

\footnotetext{
15 This is the sum of discretized profit fee difference of two marketplaces: $(0.3-0.0)+(0.3-0.1)+(0.4-0.2)+(0.5-0.3)+$ $(0.5-0.4)+(0.6-0.5)+(0.7-0.6)$. Note that the size of the lock-in region is only meaningful when competing marketplaces charge the same type of fees. It makes no sense when different types of fees are charged since they have different scales. Therefore, in the analysis of the lock-in region, we only look at the cases where marketplaces charge the same type of fees.

16 When $a^{b}=a^{s}=0.75$, rich buyers will bid 4.5 , poor buyers will bid 3 , rich sellers will ask 1.5 and poor sellers will ask 3 . When a symmetric demand and supply is considered, poor traders still can trade. However, when $a^{b}=a^{s}<0.75$, i.e. traders shade their offers more, poor traders will not be able to trade, which is irrational and they will not shade their offers at this degree. Therefore, we consider that bid factor is in the range of 0.75 to 1 .
} 


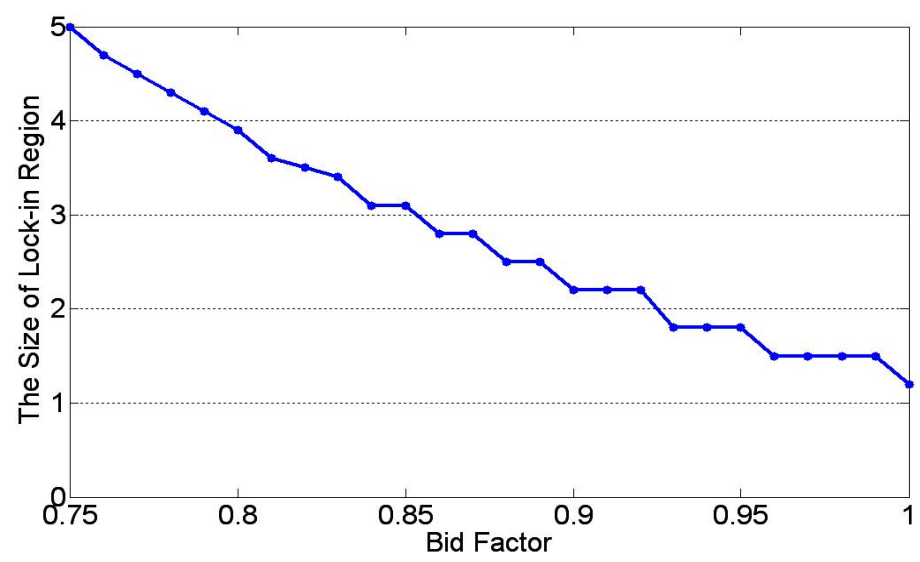

Fig. 6: Relationship between the bid factor and the size of lock-in region.

region is shown in Figure 6, from which, we can see that, when the bid factor decreases (i.e. traders shade more), the size of the lock-in region increases. This is because, when traders shade more, the absolute payments incurred from profit fees are smaller, and thus traders are less sensitive to profit fees, and therefore the size of the lock-in region increases. We also analyse the relationship between the bid factor and the size of the lock-in region when both competing marketplaces only charge registration fees. In contrast to the conclusion when both marketplaces only charge profit fees, we find that the bid factor cannot affect the size of the lock-in region significantly since the absolute payments incurred from registration fees are not affected by the bid factor.

So far, we assumed that traders always evolve from their current market selection strategies. Now we analyse how the lock-in region will be affected when some traders are able to explore other marketplaces randomly. We do this because, first of all, traders usually have incomplete information about other traders' market selection decisions. Thus they need to explore and try different marketplaces to obtain more information. This is also why in the CAT competition a similar strategy is used, where traders use $\epsilon$-greedy to randomly explore marketplaces with a small probability. Secondly, in reality, not all traders are (fully) rational, i.e. they may not always choose the cheapest marketplace. Thus we consider the case where some traders randomly select marketplaces. For example, when the randomisation probability is $10 \%$, then traders will have $90 \%$ probability of using their current market selection strategies and $10 \%$ probability of selecting each marketplace with equal probability to explore other marketplaces. For this setting, we analyse how the probability of randomisation affects the size of lock-in region. We assume that traders bid truthfully. The relationship between the randomisation probability and the size of lock-in region is shown in Figure 7. We can see that, when the probability of randomly selecting marketplace increases, the size of lock-in region decreases. Furthermore, when the probability of randomly selecting marketplaces is higher than $70 \%$, the lock-in region disappears completely. ${ }^{17}$ This means that, as exploration increases, it is more difficult for the competing marketplace to keep traders when charging higher fees even though it initially has a larger market share. ${ }^{18}$ Thus in the

\footnotetext{
17 Although a very high random exploration probability is unrealistic, here we want to analyse how the randomisation probability can affect the size of the lock-in region in the extreme case.

18 The same conclusion also holds when both competing marketplaces only charge registration fees.
} 


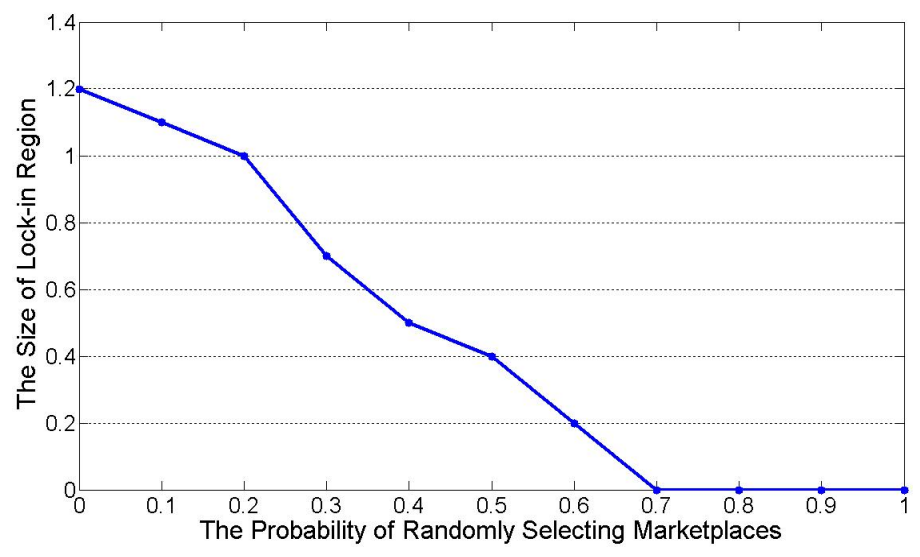

Fig. 7: Relationship between the probability of randomly selecting marketplaces and the size of lock-in region.

environment with traders having greater probabilities to explore to search for the cheaper marketplace, the marketplace with a large market share has only a limited advantage.

\subsubsection{Greater Numbers of Competing Marketplaces}

So far we have analysed the market selection strategies of traders in the setting with two competing marketplaces. As stated previously, this analysis is in line with all previous theoretical work which has focused on this canonical case, see Section 2. However, in the real world, it is often the case that more than two marketplaces compete. Now, we expect that our results will carry over to this more complex setting. Specifically, when multiple competing marketplaces only charge the same type of fees (i.e. registration or profit fees), we expect that traders will still converge to one marketplace in equilibrium. On the other hand, when multiple competing marketplaces charge different types of fees (i.e. some marketplaces charge profit fees, and others charge registration fees), we believe that traders will either converge to only one marketplace, or only two marketplaces where one charges a profit fee and the other charges a registration fee. To explore these hypotheses, we run experiments with larger numbers of marketplaces. By so doing, we find that, consistent with the previous analysis, when all marketplaces charge profit fees (or registration fees), traders will converge to one of them in equilibrium. ${ }^{19}$ Exactly which one depends on the initial starting point and market fees. Moreover, consistent with the previous analysis, in such experiments, we also find that traders may converge to the expensive marketplace in equilibrium when this marketplace initially has a larger market share. For example, when there are three competing marketplaces where marketplaces 1, 2 and 3 charge $10 \%, 20 \%$ and 30\% profit fees respectively, and initially rich traders choose marketplaces 1, 2 and 3 with probabilities $0.1,0.2$ and 0.7 respectively, and poor traders choose marketplaces 1,2 and 3 with probabilities $0.3,0.2$ and 0.5 respectively, the evolutionary results are shown in

\footnotetext{
19 This means that multiple marketplaces cannot co-exist when they charge the same type of fees. This conclusion is different from what we observe in practice. We believe that this is because in our model, different marketplaces adopt the same mechanism and have identical goods, and thus cannot provide enough diversity for traders to select different marketplaces. Furthermore, our conclusion is different from the result in Cai et al (2008), which showed that in the context of CAT competition, multiple marketplaces can retain traders when they charge similar fees. We believe that this may be because traders in the context of CAT competition adopt heuristic market selection strategies, which are different from the equilibrium strategies used in our model, and it may also be caused by the differences between our model and the context of CAT competition.
} 
Figure 8(a). From the evolutionary results, we can see that eventually all traders converge to one marketplace. Specifically, in this case, traders converge to marketplace 3 which is the most expensive since initially this marketplace has a larger market share. Furthermore, when some of them charge registration fees and others charge profit fees, we find that traders either converge to one marketplace in equilibrium or the rich traders converge to the marketplace which charges a registration fee and the poor traders converge to the marketplace which charges a profit fee (multiple competing marketplaces which charge the same type of fees do not co-exist in equilibrium and only one of them can survive). For example, when there are three competing marketplaces where marketplaces 1 and 2 charge $40 \%$ and $50 \%$ profit fees respectively and marketplace 3 charges a 0.8 registration fee, and initially rich traders choose marketplaces 1,2 and 3 with probabilities $0.15,0.2$ and 0.65 respectively, and poor traders choose marketplaces 1,2 and 3 with probabilities $0.35,0.25$ and 0.4 respectively, the evolutionary results are shown in Figure 8(b). From this figure, we can see that eventually rich traders converge to marketplace 3 charging a registration fee, and poor traders converge to marketplace 1 charging a profit fee. We also can see that marketplaces 1 and 2 charging the same types of fees cannot co-exist, and only marketplace 1 survives. In these experiments, we assume that traders bid truthfully. However, when traders can shade their offers, we still find the similar conclusions.

\section{Equilibrium Analysis of Fee Strategies}

In the previous section, we analysed the traders' equilibrium strategies of market selection for given fee systems. Now, given insights of the above analysis, we analyse how marketplaces should set fees to make profits. That is, we consider the Bayes-Nash equilibrium of the entire game, including the strategies of the marketplaces. As we said previously, this is a two-stage game, where in the first stage, marketplaces publish their fee structures according to their (mixed) fee strategies, and in the second stage, traders select marketplaces according to their market selection strategies, which are conditional on the fee system. However, given this complicated setting of a two-stage game with incomplete information about traders' types, it is difficult to use traditional game-theoretic methods to analyse equilibrium fee strategies. Intuitively, we can see that traders' market selection strategies and marketplaces' fee strategies will affect each other. Hence we use a co-evolutionary approach to analyse this problem. This co-evolutionary approach can capture the dynamic process of how marketplaces evolve their fee strategies to converge to equilibrium while taking into account the dynamic changes of the traders' market selection strategies. ${ }^{20}$ In the following, before we perform the co-evolutionary analysis, we first describe the co-evolutionary process in detail.

\subsection{The Co-Evolutionary Process}

In the co-evolutionary process, both competing marketplaces and traders dynamically learn to adapt their strategies to maximise their own expected utilities. This learning process is repeated until both traders and marketplaces do not change their strategies. At this moment, an equilibrium is reached. In each learning round (i.e. a co-evolutionary step), traders update their expected utilities before they evolve their market selection strategies. Now in order

\footnotetext{
20 Cliff (2002) and Phelps et al (2001) used genetic programming based approaches to analyse traders' bidding behaviour and the marketplace's mechanism in a co-evolutionary way. However, their work focused on isolated marketplaces without considering competition between them.
} 


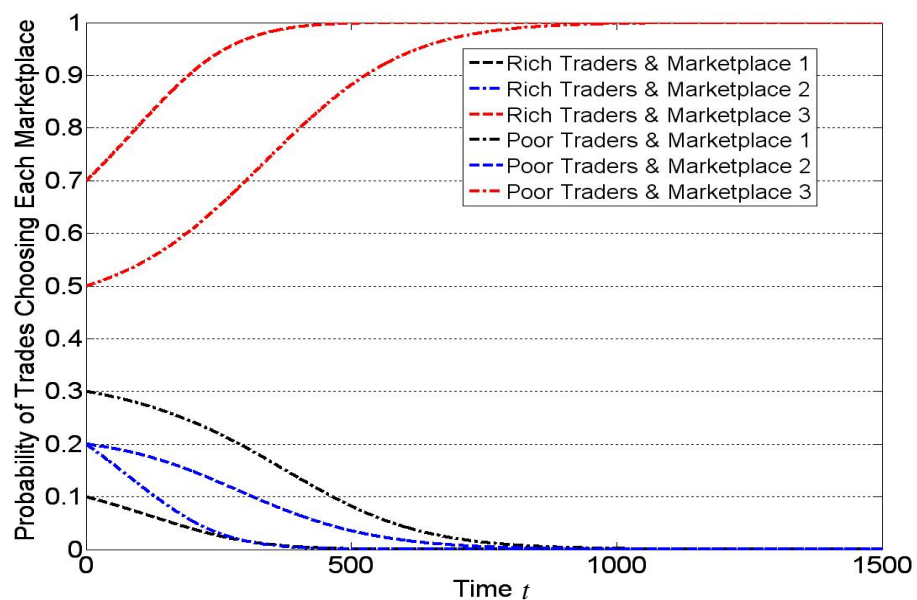

(a) Marketplace 1, 2 and 3 charge 10\%, 20\% and 30\% profit fees respectively.

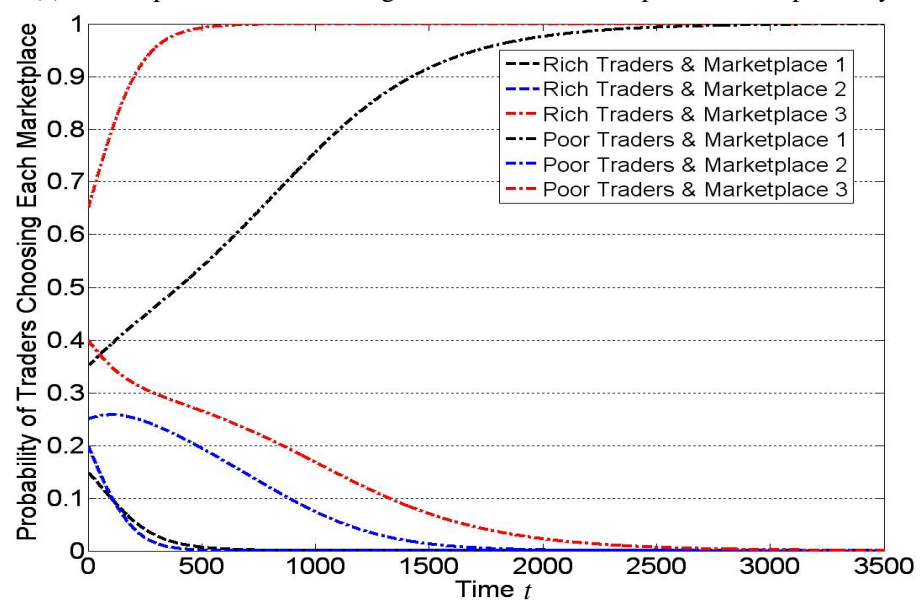

(b) Marketplace 1, 2 and 3 charge $40 \%, 50 \%$ profits fees and 0.8 registration fee respectively.

Fig. 8: Evolutionary process of market selection strategies with 3 competing marketplaces. Traders bid truthfully, i.e. $a^{b}=a^{s}=1$.

to calculate the expected utilities, they require information about bid and ask distributions, which, in turn, depend on the fees charged by the marketplaces as well as the traders' market selection strategies. While in Section 4 the bid and ask distributions were calculated for a given fee system, the strategies of the marketplaces are mixed and therefore in this case we calculate the distributions based on the composition of the marketplace strategies. However, we should note that the market selection strategies of traders are still conditional on the actual fee system announced by the marketplaces. This is important since it creates a link between the strategy composition of the marketplaces and its effect on the expected utility of the traders, enabling co-evolution to occur. ${ }^{21}$

21 An alternative approach is to keep the bid and ask distributions conditional on the fees, but then the population dynamics of the fee strategies will have no effect on the traders' expected utilities. 


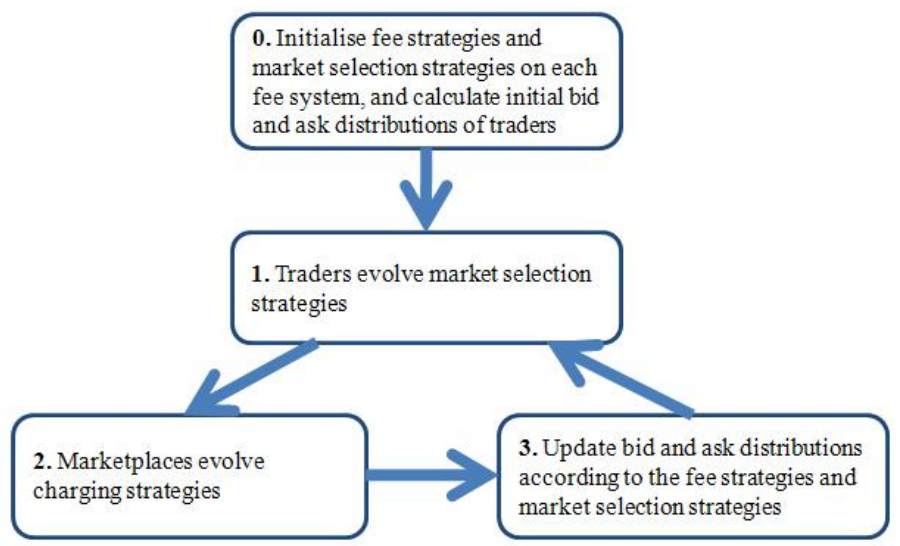

Fig. 9: The co-evolutionary process.

Now we describe the co-evolutionary process in detail (see Figure 9). First, we initialise the marketplaces' fee strategies and the traders' market selection strategies. Then we calculate the initial bid distribution of buyers and ask distribution of sellers (see Equations 21 and 22 in the following subsection). From the initial bid and ask distributions, traders calculate their expected utilities, and then evolve their market selection strategies on each possible fee system. After traders evolve their market selection strategies on each possible fee system, competing marketplaces calculate their expected utilities (which depend on the traders' conditional mixed market selection strategies) and then evolve their fee strategies. After marketplaces evolve their fee strategies, we update the bid and ask distributions of traders, and then enter the next co-evolutionary step. This co-evolutionary process proceeds until all dynamic changes of traders' market selection strategies and marketplaces' fee strategies become zero. At this moment, an equilibrium is reached.

In the following, before giving the experimental analysis in detail, we first need to derive equations to calculate the expected utilities of traders and marketplaces, and give the replicator dynamics equations.

\subsection{Expected Utilities of Traders and Marketplaces}

As per Section 4, we consider that traders adopt ray bidding strategies and marketplaces use equilibrium matching policies to match traders. As discussed above, in the co-evolutionary process, a trader's expected utility depends on the bid and ask distributions of other traders. In Subsection 4.1, we calculated the bid and ask distributions for a given fee system. However, since the fee strategies of the marketplaces are mixed, here we consider traders' offer distributions under all allowable fee systems, and therefore need to recalculate them. The new bid and ask distributions are derived in the following way respectively. First, the probability of the bid $d^{b}$ submitted by a buyer in marketplace $m$ is:

$$
\phi_{m}\left(d^{b}\right)=\sum_{\bar{P} \in \mathcal{P} M} \mu(\bar{P}) \times \phi_{m}\left(d^{b} \mid \bar{P}\right)
$$

where $\mu(\bar{P})=\prod_{m \in \mathcal{M}} \mu_{m}\left(p_{m}\right)$ is the probability of the fee system $\bar{P}$ appearing, and $\phi_{m}\left(d^{b} \mid \bar{P}\right)$ the probability of the bid $d^{b}$ submitted by a buyer in marketplace $m$ for a given fee system 
$\bar{P}$ (see Equation 6). The equations for sellers can be recalculated in the same way, which is:

$$
\phi_{m}\left(d^{s}\right)=\sum_{\bar{P} \in \mathcal{P} M} \mu(\bar{P}) \times \phi_{m}\left(d^{s} \mid \bar{P}\right)
$$

All other equations to calculate the traders' expected utilities are the same as before except that in these equations we need to replace $\phi_{m}\left(d^{b} \mid \bar{P}\right)$ and $\phi_{m}\left(d^{s} \mid \bar{P}\right)$ (which are conditional on a specific fee system $\bar{P}$ ) by $\phi_{m}\left(d^{b}\right)$ and $\phi_{m}\left(d^{s}\right)$ (which are under all possible fee systems) respectively.

In addition to the traders' utilities, in this two-stage game, we also calculate the expected utility of each marketplace. Intuitively, a marketplace's expected utility not only depends on its own fee strategy, but also on fee strategies of other marketplaces and the number of different bids and asks in it. In the following, we calculate the expected utility of marketplace $m$ given a fee strategy profile $\bar{\mu}$, and market selection strategy profiles of buyers and sellers, $\bar{\omega}^{b}(\cdot)$ and $\bar{\omega}^{s}(\cdot)$, when traders adopt ray bidding strategies. In the first step, we need to calculate the marketplace's expected utility with a fee structure $p_{m}$. In order to do this, we calculate the marketplace's expected utility when the fee system is $\bar{P}$ satisfying $p_{m}=\bar{P}(m)$, given market selection strategy profiles of buyers and sellers, $\bar{\omega}^{b}(\bar{P})$ and $\bar{\omega}^{s}(\bar{P})$. Since the marketplace's expected utility also depends on the bids and asks in this marketplace, we first use a $\mid D^{b}$-tuple $\bar{x}^{b}=\left\langle x_{1}^{b}, \ldots, x_{\left|D^{b}\right|}^{b}\right\rangle \in \mathcal{X}^{b}, \sum_{i=1}^{\left|D^{b}\right|} x_{i}^{b} \leq B$, to represent the number of different bids in marketplace $m$, where $x_{i}^{b}$ is the number of buyers submitting bid $d_{i}^{b} \in D^{b}$ in marketplace $m$ (note that this is also the number of buyers having type $\theta^{b}$ and choosing marketplace $m$ where $d_{i}^{b}=\eta^{b}\left(\theta^{b}\right)$ ), and $\mathcal{X}^{b}$ is the set of all such possible tuples. Similarly, we use a $\left|D^{s}\right|$-tuple $\bar{x}^{s}=\left\langle x_{1}^{s}, \ldots, x_{\left|D^{s}\right|}^{s}\right\rangle \in \mathcal{X}^{s}$ to represent the number of different asks in marketplace $m$. Now given the number of different bids and asks in marketplace $m$, marketplace $m$ 's expected utility is calculated as follows. Since marketplace $m$ uses equilibrium matching to match traders, we first sort the bids descendingly and asks ascendingly in marketplace $m$, and then match high bids with low asks. Specifically, we assume that there are $T$ transactions in total in marketplace $m$, and in transaction $t$, we use $\mathrm{TP}_{t}, \Lambda_{t}^{b}$ and $\Lambda_{t}^{s}$ to represent the transaction price, the buyer's observed profit, and the seller's observed profit respectively. These can be easily calculated. For example, for a transaction made by buyer with bid $d_{t}^{b}$ and seller with ask $d_{t}^{s}$, the transaction price is $\mathrm{TP}_{t}=d_{t}^{s} \times k_{m}+d_{t}^{b} \times\left(1-k_{m}\right)$, the buyer's observed profit is $\Lambda_{t}^{b}=d_{t}^{b}-\mathrm{TP}_{t}=\left(d_{t}^{b}-d_{t}^{s}\right) \times k_{m}$, and the seller's observed profit is $\Lambda_{t}^{s}=\mathrm{TP}_{t}-d_{t}^{s}=\left(d_{t}^{b}-d_{t}^{s}\right) \times\left(1-k_{m}\right)$. The marketplace's utility is:

$$
U_{m}\left(\bar{P}, \bar{x}^{b}, \bar{x}^{s}\right)=\sum_{i=1}^{\left|D^{b}\right|} x_{i}^{b} \times r_{m}+\sum_{i=1}^{\left|D^{s}\right|} x_{i}^{s} \times r_{m}+\sum_{t=1}^{T}\left(\Lambda_{t}^{b} \times q_{m}+\Lambda_{t}^{s} \times q_{m}\right)
$$

where the former two parts are profits from charging registration fees to buyers and sellers respectively, and the last part is the profit from charging profit fees.

Now we have obtained the marketplace's expected utility given the number of different bids and asks, $\bar{x}^{b}$ and $\bar{x}^{s}$. Furthermore, the probability of $\bar{x}^{b}$ appearing given market selection strategy profiles of buyers and sellers in the fee system $\bar{P}, \bar{\omega}^{b}(\bar{P})$ and $\bar{\omega}^{s}(\bar{P})$, is:

$$
\varrho_{m}^{b}\left(\bar{x}^{b}, \bar{\omega}^{b}(\bar{P}), \bar{\omega}^{s}(\bar{P})\right)=\left(\begin{array}{c}
B \\
x_{1}^{b}, \ldots, x_{\left|D^{b}\right|}^{b}
\end{array}\right) \times \prod_{i=1}^{\left|D^{b}\right|}\left(\phi_{m}\left(d_{i}^{b} \mid \bar{P}\right)\right)^{x_{i}^{b}} \times\left(1-\sum_{i=1}^{\left|D^{b}\right|} \phi_{m}\left(d_{i}^{b} \mid \bar{P}\right)\right)^{B-\sum_{i=1}^{\left|D^{b}\right|} x_{i}^{b}}
$$


Recall that $\phi_{m}\left(d_{i}^{b} \mid \bar{P}\right)$ is the probability of a buyer submitting bid $d_{i}^{b}$ in marketplace $m$ given the fee system $\bar{P}$. The probability of $\bar{x}^{s}$ appearing is:

$$
\varrho_{m}^{s}\left(\bar{x}^{s}, \bar{\omega}^{b}(\bar{P}), \bar{\omega}^{s}(\bar{P})\right)=\left(\begin{array}{c}
S \\
x_{1}^{s}, \ldots, x_{\left|D^{s}\right|}^{s}
\end{array}\right) \times \prod_{i=1}^{\left|D^{s}\right|}\left(\phi_{m}\left(d_{i}^{s} \mid \bar{P}\right)\right)^{x_{i}^{s}} \times\left(1-\sum_{i=1}^{\left|D^{s}\right|} \phi_{m}\left(d_{i}^{s} \mid \bar{P}\right)\right)^{S-\sum_{i=1}^{\left|D^{s}\right|} x_{i}^{s}}
$$

At this moment, we can get the marketplace's expected utility in the fee system $\bar{P}$

$$
\tilde{U}_{m}\left(\bar{P}, \bar{\omega}^{b}(\bar{P}), \bar{\omega}^{s}(\bar{P})\right)=\sum_{\bar{x}^{b} \in \mathcal{X}^{b}} \sum_{\bar{x}^{s} \in \mathcal{X}^{s}} \varrho_{m}^{b}\left(\bar{x}^{b}, \bar{\omega}^{b}(\bar{P}), \bar{\omega}^{s}(\bar{P})\right) \times \varrho_{m}^{s}\left(\bar{x}^{s}, \bar{\omega}^{b}(\bar{P}), \bar{\omega}^{s}(\bar{P})\right) \times U_{m}\left(\bar{P}, \bar{x}^{b}, \bar{x}^{s}\right)
$$

Then the marketplace's expected utility when its fee structure is $p_{m}$ is as follows:

$$
\tilde{U}_{m}\left(p_{m}, \mu_{-m}(\cdot)\right)=\sum_{\bar{P} \in \mathcal{P} M: p_{m}=\bar{P}(m)} \prod_{l \in \mathcal{M} \backslash\{m\}} \mu_{l}(\bar{P}(l)) \times \tilde{U}_{m}\left(\bar{P}, \bar{\omega}^{b}(\bar{P}), \bar{\omega}^{s}(\bar{P})\right)
$$

Then the marketplace $m$ 's expected utility with a fee strategy profile $\bar{\mu}$ is:

$$
\tilde{U}_{m}(\bar{\mu})=\sum_{p_{m} \in \mathcal{P}} \mu_{m}\left(p_{m}\right) \times \tilde{U}_{m}\left(p_{m}, \mu_{-m}(\cdot)\right)
$$

\subsection{Replicator Dynamics}

We now describe the replicator dynamics equations for traders and marketplaces respectively for the two-stage game. In addition to adding the replicator dynamics equations for the marketplaces, the two-stage game also requires a considerable increase in the number of equations for the traders. This is because, while in Section 4.2, the equations were for a given fee system, these are now conditional on the fee system, and a different population of traders evolves for each possible fee system. Specifically, when the fee system is $\bar{P}$, the replicator dynamics equations of each type of trader are given by Equations 19 and 20. Since there are $\left|\mathcal{P}^{M}\right|$ different fee systems, for each type of trader in each marketplace, there are $\left|\mathcal{P}^{M}\right|$ replicator equations. Then in our system considering 2 types of traders (rich and poor), in total, there are $\left|\mathcal{P}^{M}\right| \times M \times 2$ replicator equations for traders.

Now we describe replicator dynamics equations for the marketplaces. Since there are $|\mathcal{P}|$ allowable fee structures, for each marketplace, there are $|\mathcal{P}|$ replicator dynamics equations for its fee strategy. In total there are $M \times|\mathcal{P}|$ replicator dynamics equations for marketplaces. Specifically, marketplace $m$ 's replicator dynamics equation for fee structure $p_{m}$ is as follows:

$$
\dot{\mu}_{m}\left(p_{m}\right)=\frac{d \mu_{m}\left(p_{m}\right)}{d t}=\left(\tilde{U}_{m}\left(p_{m}, \mu_{-m}(\cdot)\right)-\tilde{U}_{m}(\bar{\mu})\right) \times \mu_{m}\left(p_{m}\right)
$$

where $\dot{\mu}_{m}\left(p_{m}\right)$ describes how the marketplace $m$ changes its probability of choosing fee structure $p_{m}, \tilde{U}_{m}\left(p_{m}, \mu_{-m}(\cdot)\right)$ is the marketplace $m$ 's expected utility of choosing fee structure $p_{m}$, and $\tilde{U}_{m}(\bar{\mu})$ is the marketplace $m$ 's overall expected utility as derived in Section 5.2. 


\subsection{Experimental Results}

After describing the co-evolutionary process and the replicator dynamics equations, we are ready to analyse how marketplaces evolve their fee strategies over time. Since our framework only considers a finite set of fees, specifically, we discretize the profit and registration fees from 0 to 1 with step size 0.1 . In the following analysis, we assume that there are 2 competing marketplaces, and other assumptions are the same as those in Section 4.2. In addition, unless mentioned otherwise, we assume that traders bid truthfully. ${ }^{22}$ We now analyse marketplaces' equilibrium fee strategies from different initial conditions. We first consider that both marketplaces can only charge the same type of fees (we choose profit fees as an example). We then extend the analysis to the case where different types of fees are allowed. ${ }^{23}$

\section{Two identical marketplaces initially having the same fee strategy:}

First, we consider that both marketplaces only charge profit fees. Then there are 11 possible fee structures $^{24}$ for each marketplace, which implies that there are 484 replicator dynamics equations for the traders and 22 replicator dynamics equations for the two competing marketplaces. We assume that initially both marketplaces are identical. That is, they have the same probabilities of choosing each fee structure, and for each fee system, the initial probability of traders choosing marketplace 1(2) is the ratio of profit fee of marketplace 2(1) to the sum of profit fees of both competing marketplaces (this means that traders have higher initial probabilities of choosing the cheaper marketplace). Then the initial probability of traders choosing each marketplace under all possible fee systems is equal, i.e. 0.5. From this setting, we evolve the fee strategies of marketplaces and the market selection strategies of the traders. The evolutionary process of fee strategies is shown in Figure 10. Note that, in this case, two initially identical marketplaces have the same evolutionary process. From the figure, we can see that during the evolutionary process, both marketplaces gradually set low fees with higher probability, and after 500 evolutionary steps, both marketplaces begin to converge to charge $10 \%$ profit fee. In equilibrium, we can see that both marketplaces set $10 \%$ profit fee with $100 \%$ probability. This is because two identical marketplaces have to undercut each other by decreasing fees to attract traders. Eventually, they converge to $10 \%$ profit fee, which is the minimum allowed profit fee which can guarantee positive profit for marketplaces. ${ }^{25}$ In addition, for the traders' evolutionary process, we look at the traders' probability of choosing marketplace 1 considering all possible fee systems. In this case, the probability of traders choosing each marketplace is unchanged, which is 0.5 . This shows that in a severely competitive environment, competing marketplaces have to charge the lowest fees, and even no fees, in order to keep traders. When we consider the case where traders can shade their offers, we still find that eventually marketplaces charge the minimum allowed profit fee (i.e. $10 \%$ profit fee in this setting). Furthermore, when both marketplaces initially have the same fee strategy only charging registration fees, we find that both marketplaces charge 0.1 registration fee in equilibrium.

In the previous analysis (see Section 4.2.2), we introduced randomisation for the traders' market selection strategies to analyse the effect of exploration and bounded rationality. Now

\footnotetext{
22 The equilibrium analysis in the cases where traders shade their offers is similar.

23 In the analysis of marketplaces' fee strategies, in order to reduce the fee system space, we assume that each marketplace can only charge one type of fees. We believe that our conclusions based on this assumption are still valid in the general cases. In the future work, we would like to demonstrate this by considering that marketplaces can charge arbitrary combinations of fees.

24 They are $(0,0),(0,0.1),(0,0.2),(0,0.3),(0,0.4),(0,0.5),(0,0.6),(0,0.7),(0,0.8),(0,0.9),(0,1.0)$.

25 If a lower minimal profit fee would have been allowed, e.g. $1 \%$, then both competing marketplaces will converge to this lower profit fee.
} 


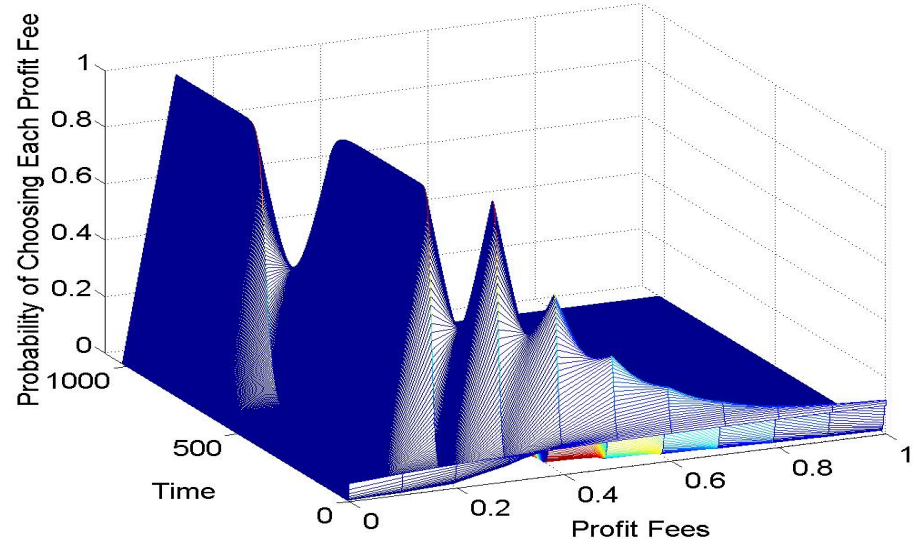

Fig. 10: Evolutionary process of fee strategies of marketplace 1 and 2 when they have identical initial fee strategies.

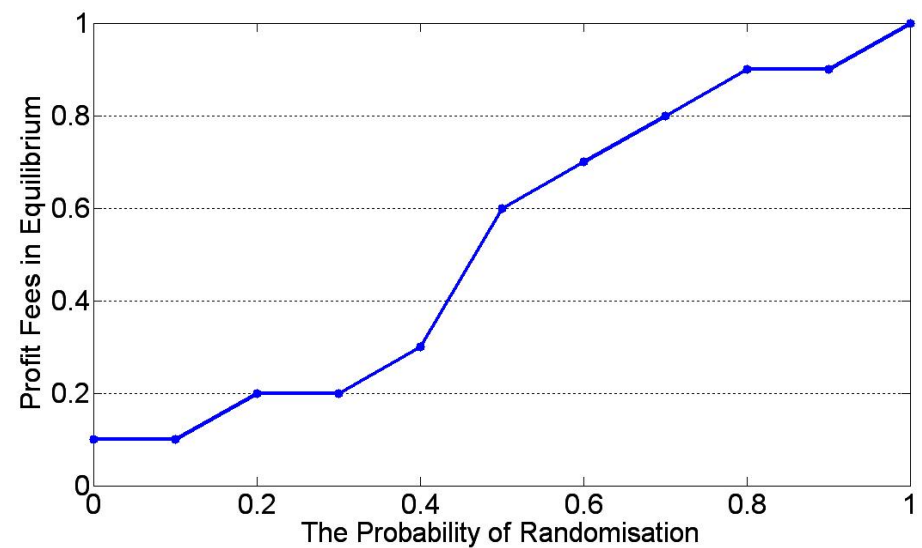

Fig. 11: Equilibrium fee strategies of marketplace 1 and 2 with respect to randomisation of market selection when two competing marketplaces have identical initial fee strategies.

we do the same for the above co-evolutionary setting. We also consider a big range of random exploration probability (from 0 to $100 \%$-the extreme case), and in doing so, we find that, as the probability of traders randomly choosing marketplaces increases, in equilibrium, fees increase. For example, when we introduce $20 \%$ randomisation, then in equilibrium, both marketplaces will charge $20 \%$ profit fee. The result is shown in Figure 11. From this, we can see when randomisation reaches above $50 \%$, in equilibrium, marketplaces charge very high fees. Especially, when the randomisation reaches $100 \%$, both marketplaces charge $100 \%$ profit fee. This is because two identical competing marketplaces have the same evolutionary process, and thus they cannot attract traders from each other. When traders have probabilities of randomly choosing marketplace, both marketplaces will find that even though they charge higher fees, they still keep traders. Thus marketplaces will charge higher fees to make more profits. 


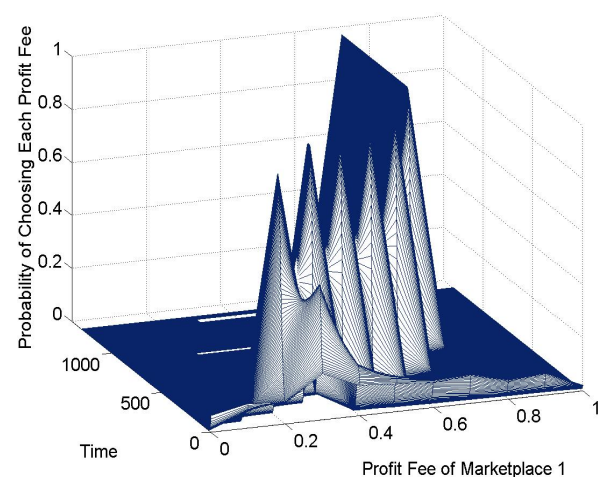

(a) Fee strategy of marketplace 1.

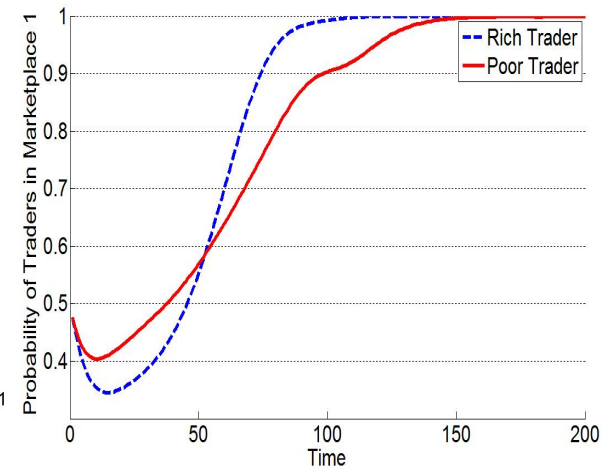

(b) Traders' probability of choosing marketplace 1 .

Fig. 12: Evolutionary process of fee strategy of marketplace 1 and traders' probability of choosing marketplace 1 when marketplace 1 adopts an adaptive fee strategy and marketplace 2 adopts a fixed fee strategy.

Two different marketplaces having an adaptive fee strategy and a fixed fee strategy respectively:

We now extend the above analysis to more general cases. In the real world, some marketplaces may adopt a fixed fee strategy, which means that they will not change fees during a specific time. To consider this situation, we now analyse how a marketplace with an adaptive fee strategy competes with a marketplace with a fixed fee strategy. As an example, we assume that marketplace 2 fixes its profit fee at $30 \%$, and marketplace 1 evolves its fee strategy and initially marketplace 1 is slightly more expensive than marketplace 2 . Therefore, marketplace 1 is slightly disadvantaged in terms of traders' probability of choosing marketplaces. The evolutionary process of the fee strategy of marketplace 1 is shown in Figure 12(a), and the dynamic changes of traders' probability of choosing marketplace 1 for this setting are shown in Figure 12(b). From these figures we can see that, initially, marketplace 1 decreases its fee (i.e. sets low fees with higher probabilities), and when it has attracted all the traders, it will increase its fee (i.e. set high fees with higher probabilities), but still keep traders. In equilibrium, marketplace 1 will charge $70 \%$ profit fee. This is because the profit fees of both competing marketplaces are within the lock-in region, and therefore marketplace 1 can keep traders even though it is more expensive. However, if in the beginning marketplace 1 is much more expensive than marketplace 2 (which means that marketplace 2 has a very large lock-in region), then even though marketplace 1 charges a very low fee, it still fails to attract traders. Therefore, if both marketplaces have similar initial fee strategies, then the marketplace using an adaptive fee strategy can beat the marketplace using a fixed fee strategy. However, when a marketplace initially has a large market share, it is difficult for a new marketplace to obtain market share, even when undercutting its competitors. We then run experiments by considering that both competing marketplaces only charge registration fees, we still find the similar results.

\section{Two different marketplaces having adaptive fee strategies:}

In the above, we find that an initially disadvantaged marketplace with an adaptive fee strategy can beat the advantaged one with a fixed fee strategy. Now we consider a more complicated case where both competing marketplaces can evolve their fee strategies. We want 
to analyse whether the initially disadvantaged marketplace can beat the advantaged one. For example, we assume that initially marketplace 2 is slightly cheaper than marketplace 1 , and thus marketplace 1 is slightly disadvantaged in terms of traders' probability of choosing marketplaces. For this setting, the evolutionary fee strategies of marketplace 1 and 2 are shown by Figures 13(a) and 13(b), and the dynamic changes of traders' probabilities of choosing marketplace 1 are shown in Figure 13(c). From these, we can see that in equilibrium, all traders converge to marketplace 1 , which is initially disadvantaged. The reason is that from Figures 13(a) and 13(b), we can see that marketplace 1 decreases fees to attract traders because of its disadvantageous position in the initial state, and marketplace 2 increases fees since it has an advantageous position in the initial state. Although there exist small fluctuations for traders' probabilities of choosing marketplaces because of the fee changes of marketplace 1 and 2, eventually all traders will converge to marketplace 1 . This shows that it is possible for an initial disadvantaged marketplace to beat an advantaged one by dynamically adapting its fees. We also find that once marketplace 1 attracts all traders, it will charge higher fees, around $70 \%$ profit fee, but still keep traders. However, if we again introduce randomisation with $20 \%$ random exploration probability (see Figure 14), we see that the behaviour of the traders' market selection changes significantly. ${ }^{26}$ In detail, we can see that marketplace 1 tries to charge a higher profit fee, but because of random exploration, traders will migrate to marketplace 2 (the cheaper marketplace). This causes marketplace 1 to reduce its fees and traders to migrate back to this marketplace. ${ }^{27}$ In fact, we observe that the strategies of the traders and marketplaces never converge to an equilibrium. However, by observing the overall evolutionary process, we still can see that, on average, marketplace 1 charges slightly higher fees than marketplace 2 . Furthermore, when both competing marketplaces can only charge registration fees, we still find that it is possible for the initially disadvantaged marketplace to beat the initially advantaged one.

\section{Two different marketplaces charging different types of fees:}

In the above analysis, we consider the cases where marketplaces charge the same type of fees. We now analyse how marketplaces evolve their fee strategies when different types of fees are charged. We assume that marketplace 1 charges a profit fee, and marketplace 2 charges a registration fee. Note that because of different scales between registration and profit fees, we just simply assume that initially marketplaces set each possible fee with an equal probability and traders select each marketplace with an equal probability. Now the co-evolutionary results are shown in Figure 15. We find that traders eventually converge to the same marketplace, which is in contrast to the previous analysis that when marketplaces charge different types of fees, rich traders prefer marketplaces that charge registration fees, and poor traders prefer marketplaces that charge profit fees (see Section 4.2.2). In more detail, from Figure 15(c), we find that, initially, rich traders still prefer marketplace 2 charging a registration fee, and poor traders prefer marketplace 1 charging a profit fee, which is the same as the previous analysis. However, from Figures 15(b) and 15(c), we can see that when rich traders choose marketplace 2 , this marketplace charges a higher registration fee, which drives rich traders to leave marketplace 2 . All traders eventually converge to marketplace 1. Once all traders choose marketplace 1, from Figure 15(a), we can see marketplace 1 charges $90 \%$ profit fee, but still keeps traders. Note that when running experiments from other initial

\footnotetext{
26 When the randomisation probability is less than $10 \%$, we find that traders' behaviour does not change significantly. Therefore, here we look at $20 \%$ randomisation probability in order to show the changes of traders' behaviour when it is affected by the random exploration significantly.

27 This is similar to the work done by Greenwald and Kephart (1999), where by adopting the derivative-following strategy, the seller continues to increase its fees until it finds that its profit falls. Then the seller begins to decrease its fees.
} 


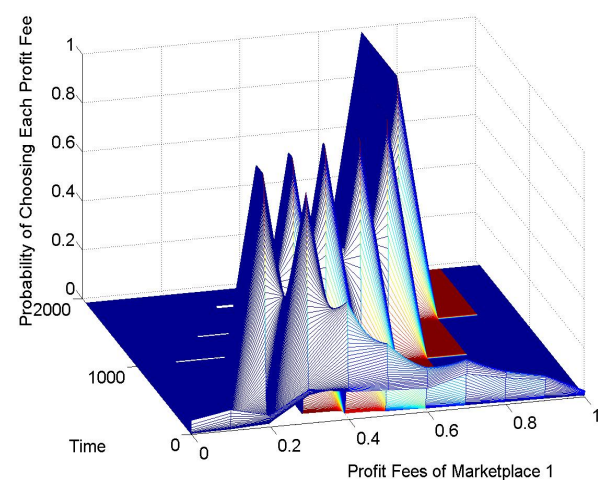

(a) Fee strategy of marketplace 1

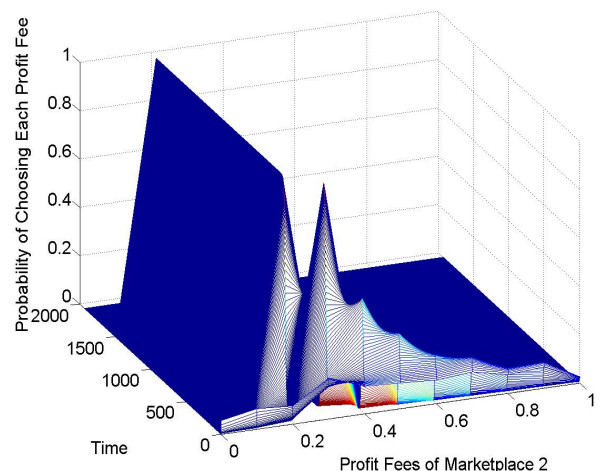

(b) Fee strategy of marketplace 2 .

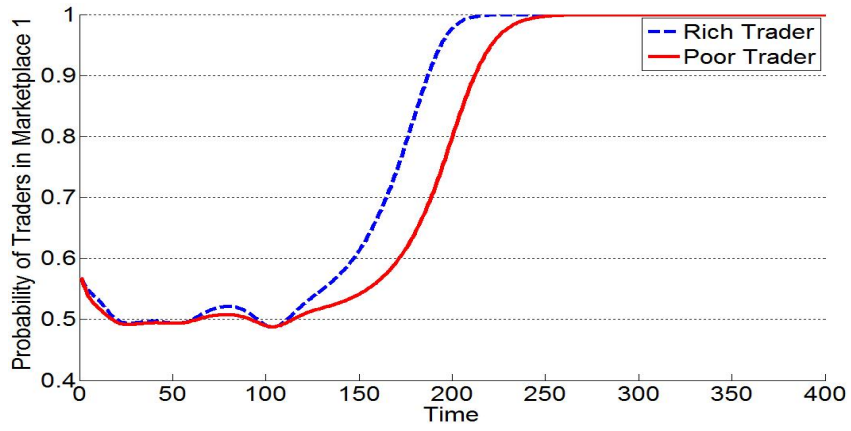

(c) Traders' probability of choosing marketplace 1.

Fig. 13: Evolutionary process of fee strategies of marketplace 1 and 2 and traders' probability of choosing marketplace 1 when two competing marketplaces have different initial fee strategies.

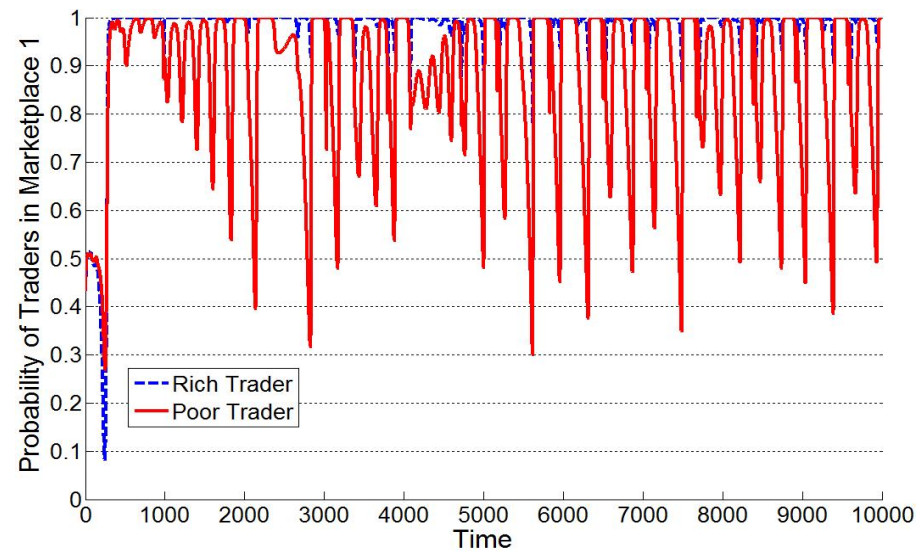

Fig. 14: Traders' probability of choosing marketplace 1 with $20 \%$ random exploration when two competing marketplaces have different initial fee strategies . 


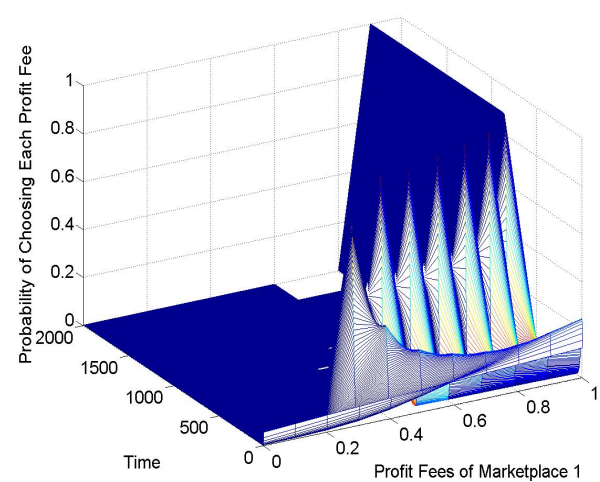

(a) Fee strategy of marketplace 1

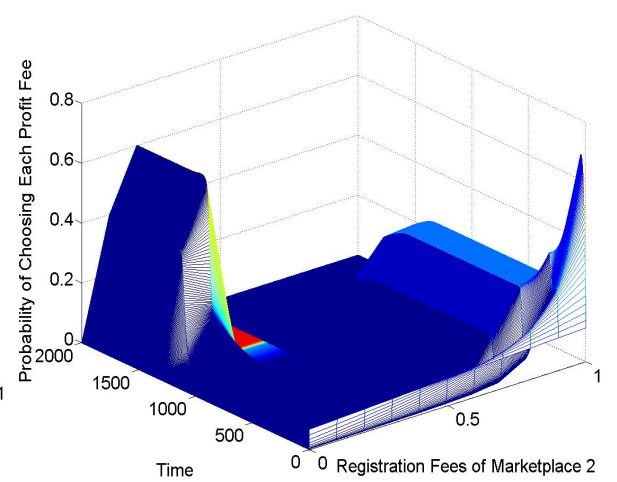

(b) Fee strategy of marketplace 2.

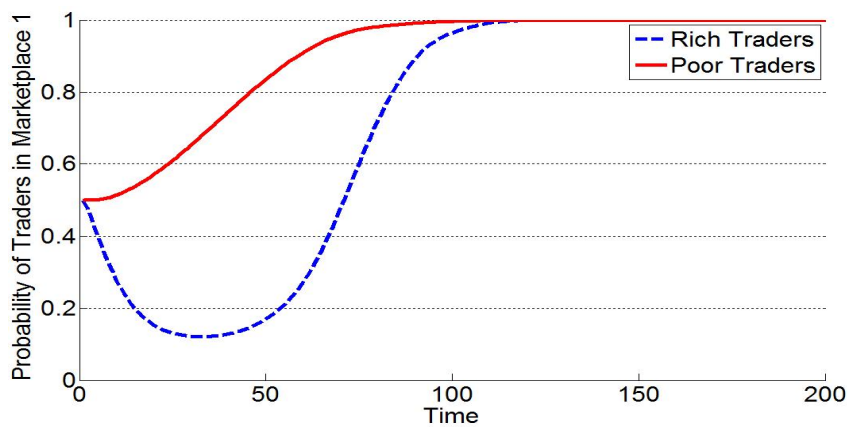

(c) Traders' probability of choosing marketplace 1.

Fig. 15: Evolutionary process of fee strategies of marketplace 1 and 2 and traders' probability of choosing marketplace 1 when marketplace 1 charges a profit fee and marketplace 2 charges a registration fee.

conditions, it may happen that eventually all traders converge to the marketplace charging a registration fee, and when attracting all traders, the marketplace charges a high registration fee.

\section{Conclusions}

In this paper, we have proposed a game-theoretic framework for analysing competing double auction marketplaces. Based on this framework, we then analysed the equilibrium market selection strategies for traders and fee strategies for marketplaces. We undertook the analysis under the assumptions that there are two types of traders (rich and poor), and rich buyers and rich sellers have the same learning behaviour and poor buyers and poor sellers have the same learning behaviour. Although this is clearly a simplified setting, it is in line with existing theoretical analysis in this broad area and is warranted because it sets the foundation for subsequent analysis where these restrictions can gradually be relaxed.

More specifically, we first analysed the equilibrium behaviour of the traders' market selection strategies for different fee systems. By so doing, we found that in our framework, traders will converge to one marketplace in equilibrium when the same type of fees are 
charged. However, when different types of fees are allowed, this strong market differentiation can cause co-existence of competing marketplaces in equilibrium, where rich traders prefer the marketplaces charging registration fees, and poor traders prefer the marketplaces charging profit fees. Furthermore, we found that it is possible for a competing marketplace to keep traders even when charging high fees if it already has a large market share. However, when a small proportion of the traders randomly explore various marketplaces, it is more difficult for the expensive marketplace to keep its traders. Furthermore, we also found that when traders can shade their offers, it is easier for the expensive marketplace to keep traders when both competing marketplaces can only charge profit fees. Following this, we used a co-evolutionary approach to analyse equilibrium fee strategies of marketplaces while taking into account the dynamic changes of the traders' market selection strategies. In doing so, we found that, when both competing marketplaces are identical, they will charge the minimal fee which can guarantee positive market profits and traders select them with equal probability in equilibrium. Furthermore, we found that by evolving the fee strategy, an initially disadvantaged marketplace can beat an initially advantaged one with a fixed strategy, or even also with an evolving fee strategy. We also showed that, when introducing random exploration, the strategies of the traders and marketplaces will not always converge, but the fees and movement of traders keep fluctuating. In addition, when different types of fees are allowed, different types of traders will prefer different marketplaces in the initial evolutionary steps. However, as the evolutionary process proceeds, eventually all traders will converge to one marketplace, and then this marketplace charges a high fee. When taken together, the insights of our work will be useful to help to understand how traders select a marketplace, and help to design a fee strategy for a competing marketplace. Specifically, they can be used by entrants to design and analyse key facets of competing marketplaces for the CAT competition and by enterprises running marketplaces to set fees in real-world economic activity.

There are still a number of avenues for future work. First, in this paper, we assume that traders use ray bidding strategy with fixed bid factors. While this offers important insights into the fundamental nature of behaviour, we would like to see how traders evolve their ray bidding strategies. In doing so, we are interested in analysing how market fees affect traders' equilibrium bidding strategies and how marketplaces set fees when traders adopt equilibrium bidding strategies. Second, in addition to considering ray bidding strategies, we also would like to extend our framework in order to incorporate more advanced bidding strategies to determine whether the same broad sets of behaviour are observed. Third, at present, our theoretical analysis is limited to two types of traders. In the future, we would like to extend our analysis to the case with more trader types and larger numbers of marketplaces. This will enable us to model a wider range of real-world phenomena. Finally, at present, we have assumed that the competing marketplaces use the same matching technology (i.e. they all use the equilibrium matching policy). However, in practice, they may use different technologies, as we see in the CAT competition, and we intend to analyse this using a similar approach as we have done in this paper.

Acknowledgements This paper has been significantly extended from a preliminary version that was published previously (Shi et al, 2010b).

\section{A Additional Experiments}

In this appendix we present additional experimental results for those in Section 4.2.2 by considering different starting points and different possible fee systems. We omit them from the main text because they provide no 
new insights, but are nevertheless useful in providing further support for the claims in Section 4.2.2. Specifically, we show results with all fee systems, where we discretize the profit and registration fees from 0 to 1 with step size 0.1 . For these settings we show the probability of traders choosing each marketplace in equilibrium. In more detail, for a given fee system, traders evolve from different starting points, from which we can obtain the basin of attraction to each equilibrium. As we mentioned in Section 4.2.1, given the assumption that each starting point is selected by traders with an equal probability, the size of the basin can be used as an indicator of the probability of traders converging to that equilibrium (Tuyls and Parsons, 2007). By approximating the size of each basin, we can estimate the probability of traders converging to each equilibrium. In the following figures, we use different gray levels to indicate the probability of traders choosing each marketplace in equilibrium (the darker, the higher the probability of traders choosing the marketplace indicated in the figure).

\section{A.1 Both marketplaces only charging profit fees:}

Firstly, we present additional experimental results in the case where both marketplaces only charge profit fees. For this setting we show the probability of traders choosing each marketplace in equilibrium in different combinations of profit fees (see Figure 16 for traders bidding truthfully and Figure 17 for traders shading offers). Note that in this case the probability of rich traders choosing each marketplace in equilibrium is the same as that of poor traders. From these experiments, we still find that traders always converge to the same marketplace in equilibrium. Furthermore, comparing Figures 16 with 17, we can see that traders have a slightly higher probability of choosing the expensive marketplace when traders can shade their offers than when traders bid truthfully. All of these conclusions are consistent with our previous claims made from Figures 1(a) and 1(b).

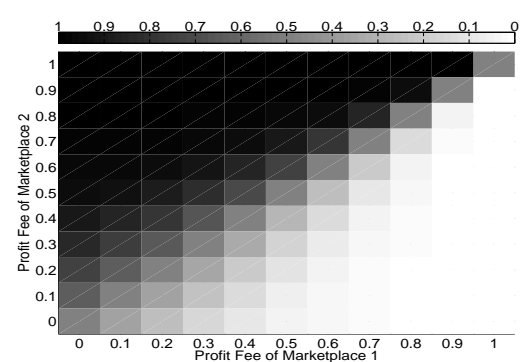

(a) The probability of choosing marketplace 1

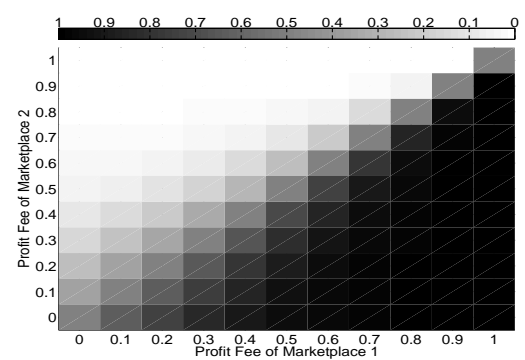

(b) The probability of choosing marketplace 2 .

Fig. 16: The probability of rich and poor traders choosing each marketplace in equilibrium. $a^{b}=a^{s}=1$.

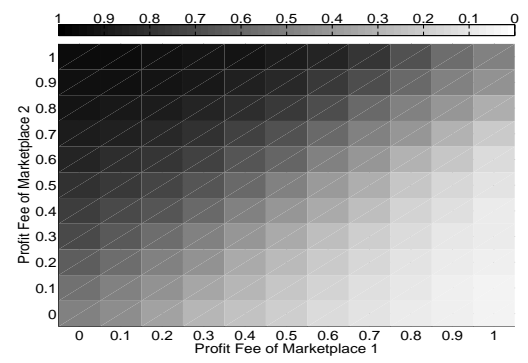

(a) The probability of choosing marketplace 1 .

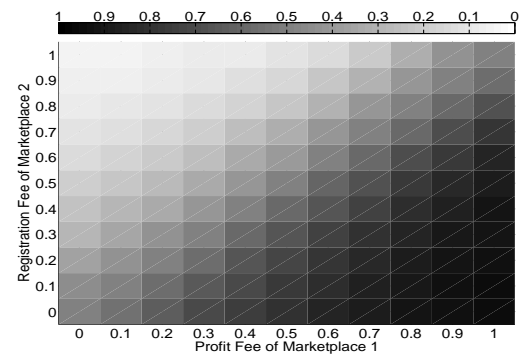

(b) The probability of choosing marketplace 2 .

Fig. 17: The probability of rich and poor traders choosing each marketplace in equilibrium. $a^{b}=a^{s}=0.8$.

\section{A.2 Both marketplaces only charging registration fees:}

We now show the additional experimental results in the case where both marketplaces only charge registration fees. We consider different combinations of registration fees, and show the results in Figures 18 and 19 where traders bid truthfully. In this case the probabilities of rich and poor traders choosing each marketplace in equilibrium are different. From these experiments, we still find that in equilibrium, traders always converge to the same marketplace, or no marketplace. Note that both rich and poor traders have a very small probability of choosing no marketplace when registration fees in both marketplaces are high. However, the probability is so small that it cannot be visualised. Therefore we omit them. These results are consistent with our previous claims made from Figures 2(a) and 2(b). Note that when traders can shade their offers, these results are not 
affected significantly since the absolute payments incurred from registration fees are not affected by shading. Therefore, we do not show the results in this case.

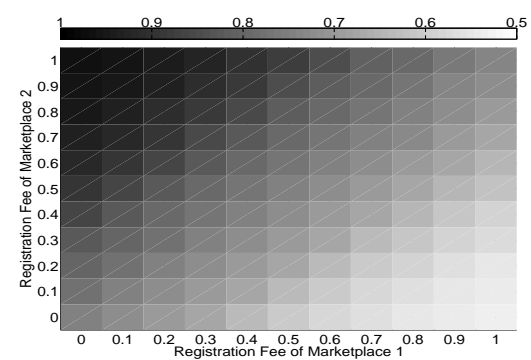

(a) The probability of choosing marketplace 1

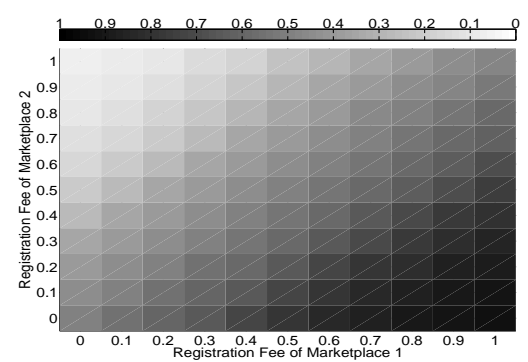

(b) The probability of choosing marketplace 2 .

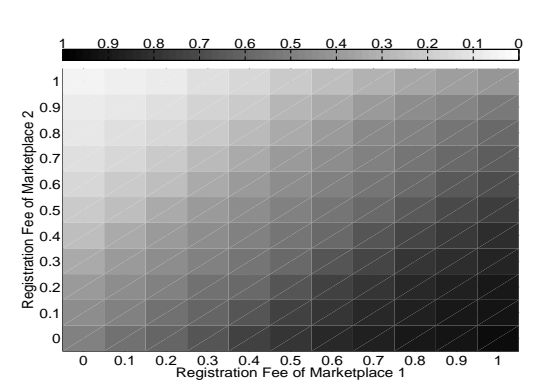

(b) The probability of choosing marketplace 2 .

(a) The probability of choosing marketplace 1

Fig. 19: The probability of poor traders choosing each marketplace in equilibrium. $a^{b}=a^{s}=1$.

A.3 Marketplace 1 charging a profit fee and marketplace 2 charging a registration fee:

Furthermore, we show the additional experimental results in the case where marketplace 1 charges a profit fee and marketplace 2 charges a registration fee. We consider different combinations of these fees, and show the results in Figures 20 and 21 where traders bid truthfully, and Figures 22 and 23 where traders shade their offers. Again we can see that the conclusions are the same as those we made in Figures 3(a) and 3(b). In more detail, comparing Figures 20 with 21, we can see that rich traders have a higher probability of choosing marketplace 2 charging a registration fee, and poor traders have a higher probability of choosing marketplace 1 charging a profit fee. Furthermore, by comparing Figures 20 and 22, and comparing Figures 21 and 23, we also can see that when traders can shade their offers, the probability of traders converging marketplace 1 charging a profit fee is increased since the payments incurred by profit fees are reduced.

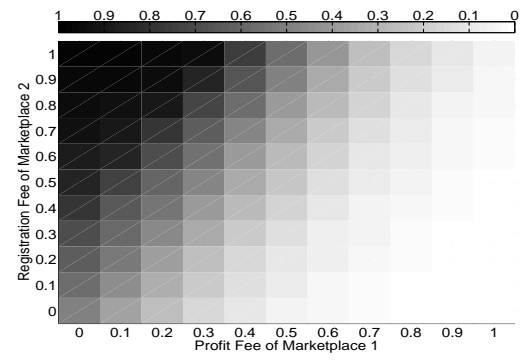

(a) The probability of choosing marketplace 1 .

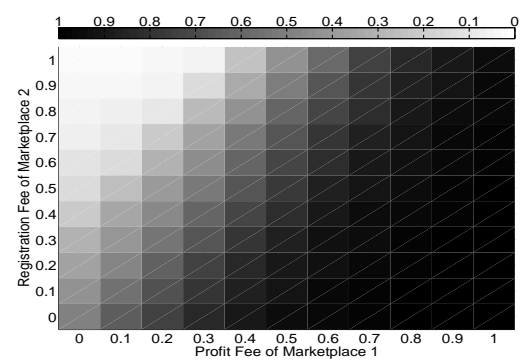

(b) The probability of choosing marketplace 2 .

Fig. 20: The probability of rich traders choosing each marketplace in equilibrium. $a^{b}=a^{s}=1$. 


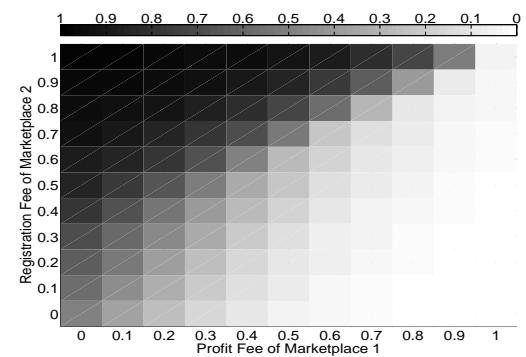

(a) The probability of choosing marketplace 1

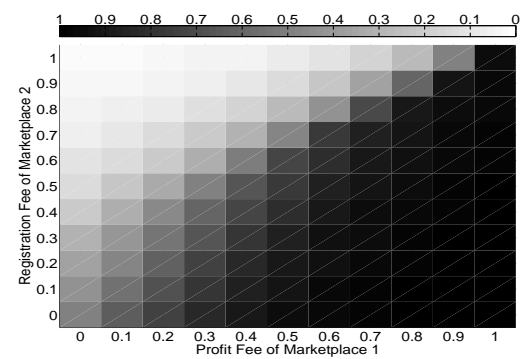

(b) The probability of choosing marketplace 2 .

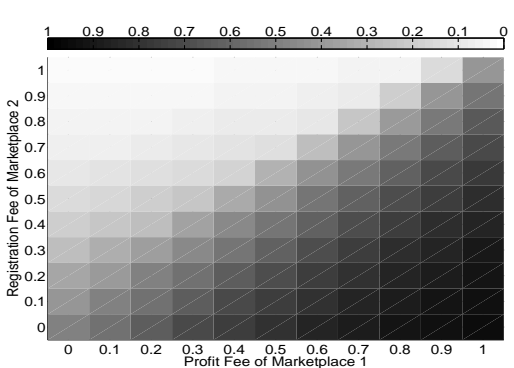

(b) The probability of choosing marketplace 2 .

(a) The probability of choosing marketplace 1

Fig. 22: The probability of rich traders choosing each marketplace in equilibrium. $a^{b}=a^{s}=0.8$.
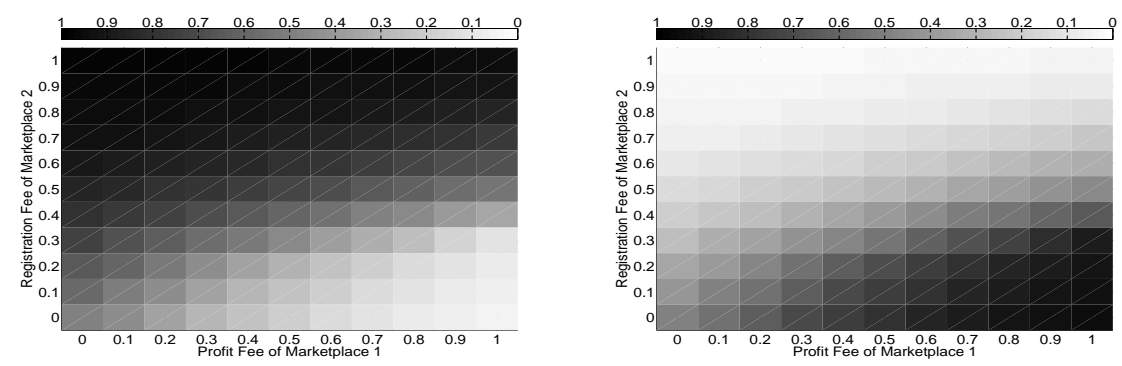

(a) The probability of choosing marketplace 1

(b) The probability of choosing marketplace 2 .

Fig. 23: The probability of poor traders choosing each marketplace in equilibrium. $a^{b}=a^{s}=0.8$.

A.4 Both marketplaces charging combinations of registration and profit fees:

Finally, we show the additional experimental results in the case where both marketplaces can charge combinations of registration and profit fees. Here we discretize registration and profit fees from 0 to 1 with step size 0.2 , instead of 0.1 , in order to reduce the fee system space. The results for different combinations of these fees are shown in Figures 24 and 25. We still find the same conclusions as those made in Figure 4, where we can see that different types of traders may converge to different marketplaces in equilibrium, and when both marketplaces charge high fees, traders may choose to visit no marketplace. 


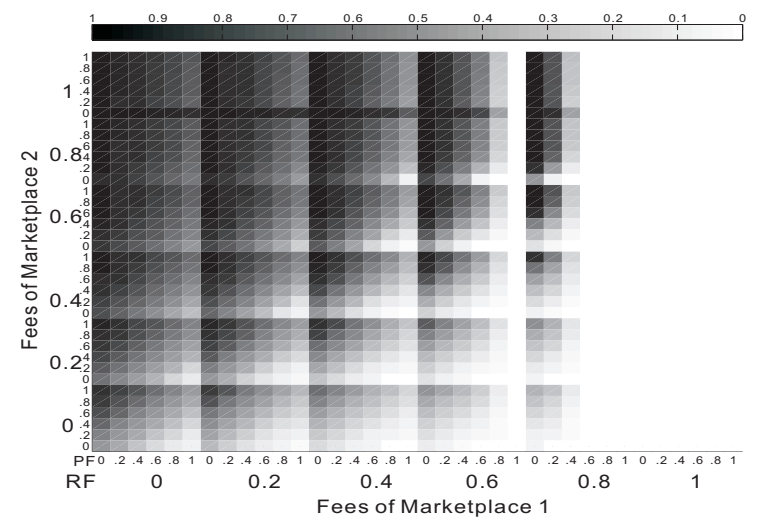

(a) The probability of choosing marketplace 1 .

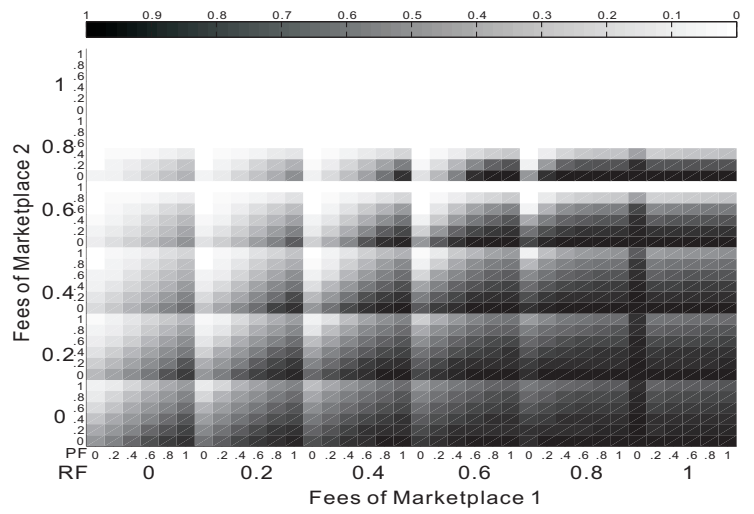

(b) The probability of choosing marketplace 2 .

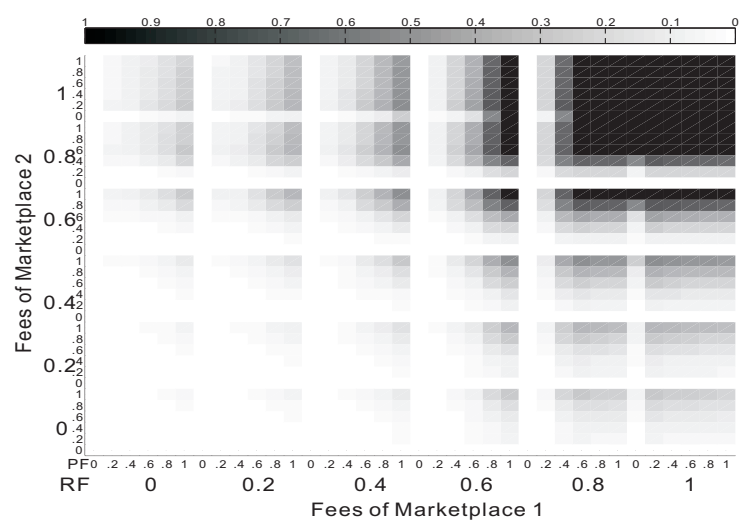

(c) The probability of choosing no marketplace.

Fig. 24: The probability of rich traders choosing each marketplace in equilibrium. $a^{b}=a^{s}=1$. $\mathrm{RF}=$ Registration Fee; $\mathrm{PF}=$ Profit Fee. 


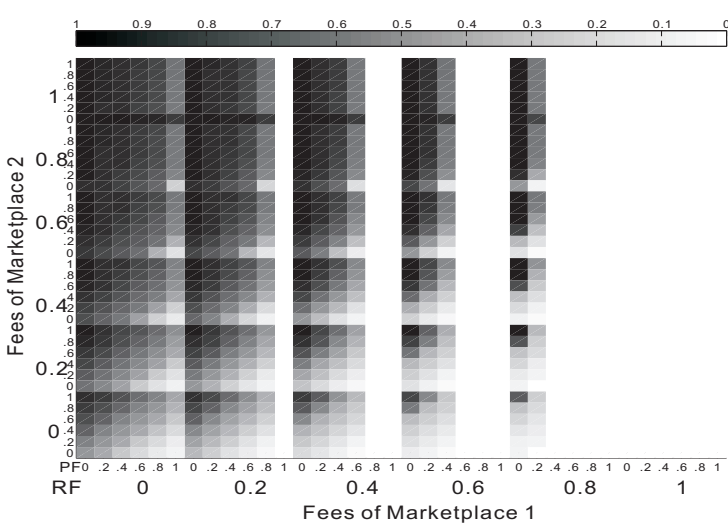

(a) The probability of choosing marketplace 1 .

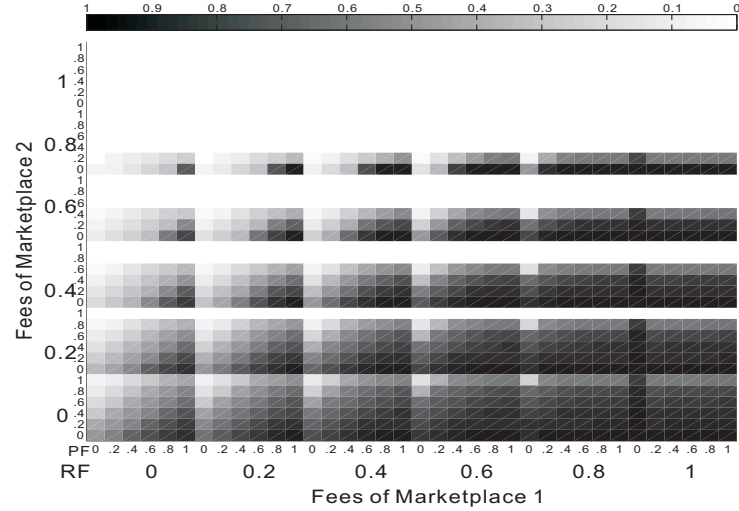

(b) The probability of choosing marketplace 2 .

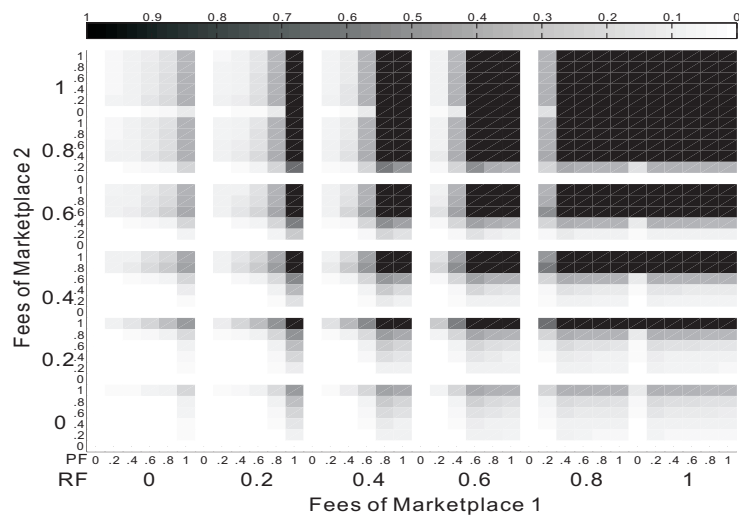

(c) The probability of choosing no marketplace.

Fig. 25: The probability of poor traders choosing each marketplace in equilibrium. $a^{b}=a^{s}=1$. $\mathrm{RF}=$ Registration Fee; $\mathrm{PF}=$ Profit Fee 


\section{References}

Anthony, P.,\& Jennings, N.R. (2006). Developing a bidding agent for multiple heterogeneous auctions. ACM Transactions on Internet Technology 3:185-217

Binmore, K., Samuelson, L.,\& Gale, J. (1995). Learning to be imperfect: The ultimatum game. Games and Economic Behavior 8(1):56-90

Burguet, R.,\& Sakovics, J. (1999). Imperfect competition in auction design. International Economic Review 40(1):231-247

Byde, A., Preist, C.,\& Jennings, N.R. (2002). Decision procedures for multiple auctions. In: Proceedings of the 1st International Joint Conference on Autonomous Agents and Multi-Agent Systems, pp 613-620

Cai, K., Niu, J.,\& Parsons, S. (2008). On the economic effects of competition between double auction markets. In: Proceedings of the 10th Workshop on Agent-Mediated Electronic Commerce

Caillaud, B.,\& Jullien, B. (2003). Chicken \& egg: Competition among intermediation service providers. RAND Journal of Economics 34(2):309-28

Cliff, D. (2002). Evolution of market mechanism through a continuous space of auction-types. In: Proceedings of the 2002 Congress on Evolutionary Computation, pp 2029-2034

Cliff, D.,\& Bruten, J. (1997). Minimal-intelligence agents for bargaining behaviors in market-based environments. Tech. rep., Hewlett Packard Research Labs

Damiano, E.,\& Hao, L. (2008). Competing matchmaking. Journal of the European Economic Association 6(4):789-818

Ellison, G., Fudenberg, D.,\& Mobius, M. (2004). Competing auctions. Journal of the European Economic Association 2(1):30-66

Farrell, J.,\& Klemperer, P. (2007). Coordination and lock-in: Competition with switching costs and network effects. In: Armstrong M, Porter R (eds) Handbook of Industrial Organization, 3:1967-2072

Friedman, D.,\& Rust, J. (1993). The double auction market: institutions, theories and evidence, vol XIV of Santa Fe Institute Studies in the Science of Complexity. Perseus Publishing

Fudenberg, D.,\& Tirole, J. (1991). Game Theory. The MIT Press

Gerding, E.H., McBurney, P., Niu, J., Parsons, S.,\& Phelps, S. (2007a). Overview of CAT: A market design competition. Tech. rep., Dept. of Computer Science, University of Liverpool

Gerding, E.H., Rogers, A., Dash, R.K.,\& Jennings, N.R. (2007b). Sellers competing for buyers in online markets: Reserve prices, shill bids, and auction fees. In: Proceedings of the 20th International Joint Conference on Artificial Intelligence, pp 1287-1293

Gjerstad, S.,\& Dickhaut, J. (1998). Price formation in double auctions. Games and Economic Behavior 22:129

Greenwald, A.,\& Kephart, J. (1999). Shopbots and pricebots. In: Proceedings of the 2nd Workshop on AgentMediated Electronic Commerce

He, M., Jennings, N.R.,\& Prugel-Bennett, A. (2006). A heuristic bidding strategy for buying multiple goods in multiple English auctions. ACM Transactions on Internet Technology 6(4):465-496

Honari, S., Ebadi, M., Foshati, A.,\& Gomrokchi, M. (2009). Price estimation of PersianCAT market equilibrium. In: Proceedings of the Workshop on Trading Agent Design and Analysis

Kartz, M.L.,\& Shapiro, C. (2007). Network externalities, competition, and compatibility. The American Economic Review 75(3):424-440

Lee, R.S. (2008). Competing platforms. Working paper

Maynard-Smith, J. (1982). Evolution and the Theory of Games. Cambridge University Press

McAfee, R.P. (1993). Mechanism design by competing sellers. Econometrica 61(6):1281-1312

Niu, J., Cai, K., Parsons, S., \& Sklar, E. (2007). Some preliminary results on competition between markets for automated traders. In: Proceedings of the Workshop on Trading Agent Design and Analysis

Niu, J., Cai, K., Gerding, E.H., McBurney, P.,\& Parsons, S. (2008). Characterizing effective auction mechanisms: Insights from the 2007 TAC Market Design Competition. In: Proceedings of the 7th International Conference on Autonomous Agents and Multi-Agent Systems, pp 1079-1086

Petric, A., Podobnik, V., Grguric, A.,\& Zemljic, M. (2008). Designing an effective e-market: an overview of the cat agent. In: Proceedings of the Workshop on Trading Agent Design and Analysis

Phelps, S., McBurney, P., Parsons, S.,\& Sklar, E. (2001). Co-evolutionary auction mechanism design: A preliminary report. In: Proceedings of the 4th Workshop on Agent-Mediated Electronic Commerce

Phelps, S., Parsons, S.,\& McBurney, P. (2006). An evolutionary game-theoretic comparison of two double auction market designs. In: Proceedings of the 6th Workshop on Agent-Mediated Electronic Commerce

Phelps, S., McBurney, P.,\& Parsons, S. (2010). Evolutionary mechanism design: a review. Autonomous Agents and Multi-Agent Systems 21(2):237-264

Redondo, F.V. (2003). Eeconomics and the Theory of Games. Cambridge University Press 
Shi, B., Gerding, E.H., Vytelingum, P.,\& Jennings, N.R. (2010a). An equilibrium analysis of competing double auction marketplaces using fictitious play. In: Proceedings of the 19th European Conference on Artificial Intelligence, pp 575-580

Shi, B., Gerding, E.H., Vytelingum, P.,\& Jennings, N.R. (2010b). A game-theoretic analysis of market selection strategies for competing double auction marketplaces. In: Proceedings of the 9th International Conference on Autonomous Agents and Multi-Agent Systems, pp 857-864

Smith, V.L. (1962). An experimental study of competitive market behavior. Journal of Political Economy 70:111-137

Sohn, J., Lee, S., \& Mullen, T. (2009). Attracting intra-marginal traders across multiple markets. In: Proceedings of the Workshop on Trading Agent Design and Analysis

Stavrogiannis, L.C.,\& Mitkas, P.A. (2009). CAT 2008 post-tournament evaluation: the Mertacor's perspective. In: Proceedings of the Workshop on Trading Agent Design and Analysis

Turocy, T.L. (2001). Computation and robustness in sealed-bid auctions. PhD thesis, Northwestern University

Tuyls, K.,\& Parsons, S. (2007). What evolutionary game theory tells us about multiagent learning. Artificial Intelligence Journal 171(7):406-416

Vytelingum, P., Cliff, D.,\& Jennings, N.R. (2008a). Strategic bidding in continuous double auctions. Artificial Intelligence Journal 172(14):1700-1729

Vytelingum, P., Vetsikas, I., Shi, B.,\& Jennings, N.R. (2008b). Iamwildcat: The winning strategy for the tac market design competition. In: Proceedings of the 18th European Conference on Artificial Intelligence, pp 428-432

Wellman, M. P. (2006). Methods for empirical game-theoretic analysis. In: Proceedings of the 21st Conference on Artificial Intelligence, pp 1552-1555

Zhan, W.,\& Friedman, D. (2007). Markups in double auction markets. Journal of Economic Dynamics and Control 31(9):2984-3005 